\title{
A STABLE ADAPTIVE NUMERICAL SCHEME FOR HYPERBOLIC CONSERVATION LAWS*
}

\author{
BRADLEY J. LUCIER $\dagger$
}

\begin{abstract}
A new adaptive finite-difference scheme for scalar hyperbolic conservation laws is introduced. A key aspect of the method is a new automatic mesh selection algorithm for problems with shocks. We show that the scheme is $L^{1}$-stable in the sense of Kuznetsov, and that it generates convergent approximations for linear problems. Numerical evidence is presented that indicates that if an error of size $\varepsilon$ is required, our scheme takes at most $O\left(\varepsilon^{-3}\right)$ operations. Standard monotone difference schemes can take up to $O\left(\varepsilon^{-4}\right)$ calculations for the same problems.
\end{abstract}

1. Introduction. Our focus in this paper is the efficient solution of the hyperbolic conservation law,

$$
\begin{aligned}
& u_{t}+f(u)_{x}=0, \quad x \in \mathbf{R}, t>0, \\
& u(x, 0)=u_{0}(x), \quad x \in \mathbf{R} .
\end{aligned}
$$

We introduce an adaptive finite-difference scheme that takes advantage of the structure of the solution of $(\mathrm{C})$ to reduce its computational complexity. We prove that the scheme is $L^{1}$ stable in the sense of Kuznetsov, and we offer numerical evidence that, because of the dynamic mesh modification, asymptotic error decay rates are improved for some problems. For linear problems we show that a version of our method converges if the initial data's first derivative is of bounded variation.

Our method is, generally speaking, in the class of viscosity methods, methods that include monotone finite-difference schemes. Monotone schemes have been analyzed by Harten et al. [13], Crandall and Majda [6], Kuznetsov [17], Sanders [22], and Lucier [19]. These schemes converge to the entropy weak solution of the conservation law (C), as formulated by Kruzkov [16]. Kuznetsov provided a general theory of approximation for approximate solutions of $(\mathrm{C})$, and he used this theory to provide error estimates for various approximation methods for $(\mathrm{C})$, including monotone difference schemes on uniform meshes. His techniques were used by Sanders and by Lucier to provide error estimates for difference schemes with nonuniform meshes and nonlocal difference operators respectively.

One of the considerations in developing our algorithm was that it must exhibit nonlinear stability properties similar to those of the differential equation itself. Solutions of $(\mathrm{C})$ are stable in $L^{1}(\mathbf{R})$ in the sense that, if $u(x, t)$ and $v(x, t)$ are solutions of $(\mathrm{C})$, then

$$
\|u(\cdot, t)-v(\cdot, t)\|_{L^{1}(\mathbf{R})} \leqq C\|u(\cdot, 0)-v(\cdot, 0)\|_{L^{1}(\mathbf{R})}
$$

for all $t>0$, with $C=1$. On the other hand, there is no $C=C(\|u(\cdot, 0)\|,\|v(\cdot, 0)\|, t)$ such that the preceding inequality holds for any $L^{p}(\mathbf{R})$ with $p>1$. In particular, since the first derivatives of $u$ are not, in general, in $L^{2}$, error analyses, such as those of Dupont [10] and Davis and Flaherty [7], that rely on the $L^{2}$ norms of the solutions of (C) may not be applied to these problems. For this reason, we have emphasized an $L^{1}$ approach in this paper.

* Received by the editors March 24, 1983, and in revised form January 10, 1984. This research was sponsored by the U.S. Army under contract DAAG29-80-C-0041. This material is based upon work partially supported by the National Science Foundation under grant MCS-7927062, Mod. 1.

† Division of Mathematical Sciences, Purdue University, West Lafayette, Indiana 47907. 
Our approach may be conceptualized as follows. Beginning with a uniform mesh in $[a, b] \times[0, T]$ with a mesh spacing of $\Delta t$, meshpoints are removed where they are not needed to achieve the required accuracy. A standard finite-difference operator is used to advance the approximate solution from one timestep to the next. (Special techniques are used when the mesh differs from one timestep to the next.) Our method differs from previous ones in the mesh selection algorithm, the interpretation of the approximate solution, and our specific choice of finite-difference operator.

Much previous work has been done in adaptive methods for evolution equations. Davis and Flaherty's adaptive method [7] is designed to solve general evolution equations, generally with smooth solutions. Our mesh selection algorithm is similar to theirs, in that it attempts to equidistribute a measure of the error among the meshpoints; the difference lies in that our algorithm equidistributes a measure of the local error in $L^{1}$ rather than $L^{2}$. Other general algorithms for evolution equations were proposed by Miller [20] and Dupont [10]. Dupont supplies convergence analyses for his methods, which are mainly finite-element algorithms with moving meshes. Gannon [12] introduced an adaptive finite-element method for parabolic differential equations based on theory for elliptic equations.

Our algorithm was also motivated by the work of Sanders [22], Douglas [8], and Douglas and Wheeler [9] on monotone finite-difference schemes with nonuniform grids. Sanders and Douglas prove convergence results for their methods on a fixed grid. Douglas and Wheeler introduce an algorithm that uses grids that may change from one timestep to the next, a true adaptive mesh method. While they do not provide error estimates, they prove that their numerical solutions converge to the entropy solution estimates, they prove that their numerical solutions converge to the entropy solution of the conservation law. We compare our method with theirs in the final section.

When Sanders, Douglas, and Douglas and Wheeler considered a nonuniform mesh, they interpreted their numerical solutions as piecewise constant in $x$, and they used a conservative finite-difference operator that is, in general, inconsistent everywhere. As a consequence, $\max \left(x_{i}-x_{i-1}\right) / \Delta t$ must be bounded to achieve stability in time in [9]. Because our mesh selection algorithm can generate arbitrarily large spatial increments, depending on the smoothness of the solution and the nonlinearity of $f$, we chose to interpret the solution as a piecewise linear function, and so to take advantage of at least some smoothness in the solution. We also use a consistent, but nonconservative, difference operator that reduces to a well-known conservative operator wherever the mesh is uniform. Because our mesh selection algorithm generates uniform spatial increments where the approximate solution lacks the requisite smoothness, the finitedifference operator is still conservative near shocks. Section 4 contains partial results that bound the global mass error in a reasonable way for piecewise smooth solutions. The numerical results of $\S 5$ show that, in actuality, the mass error behave as suggested in $\S 4$.

Adaptive numerical methods for hyperbolic conservation laws have previously been considered by Oliger [21] and his students. Hedstrom and Rodrigue [14] survey some of the techniques that are used. Bolstad [2] presents a framework for methods in one space dimension. His schemes incorporate locally varying, recursively defined space and time increments. He uses Richardson extrapolation to estimate the local truncation error of the finite-difference scheme, a quantity that determines the local grid size. Berger [21] extends Bolstad's work to two dimensions. Among other things, she deals with strictly two-dimensional problems of shock capturing, subgrid orientation, and overlapping grids.

Oliger and his students employ locally varying timesteps as well as spatial mesh increments; we do not, mainly because we were not able to prove stability and 
convergence results for the improved methods. Since asymptotic improvement in convergence rates can be exhibited with fixed (small) timesteps, locally varying temporal increments were not considered essential. When locally varying timesteps are used, our algorithm's implementation is close to Bolstad's.

The paper continues as follows. A discussion of notation concludes this section. Section 2 briefly describes the finite-difference operator used here. Section 3 presents the mesh selection algorithm, and proves certain useful properties about the resulting mesh. Section 4 contains proofs of the nonlinear stability of the algorithm. Section 5 shows that a variant of our method converges for solutions of linear problems. Section 6 details our implementation of the algorithm. And finally, $\S 7$ describes our computational results.

We will generally choose initial data from the class of functions whose value, or first derivative, is of bounded variation. The bounded-variation seminorm of a function $u \in B V(\mathbf{R})$ is defined as $|u|_{B V(\mathbf{R})}=\int_{\mathbf{R}}\left|u^{\prime}(x)\right| d x$, where the integrand is interpreted as a finite measure. If $u$ is in $B V(\mathbf{R})$, then there are two bounded functions, $u^{+}$and $u^{-}$ such that $u=u^{+}+u^{-}$and $u^{+}$is nondecreasing, $u^{-}$is nonincreasing. We define $u^{t}=$ $u^{+}-u^{-}$, the total variation function of $u$. We also define the maximum and minimum operators $u \vee v=\max (u, v)$, and $u \wedge v=\max (u, v)$.

Throughout he paper we assume the normalization that

$$
\|f\|_{\text {Lip }}=\sup _{x \neq y} \frac{|f(x)-f(y)|}{|x-y|} \leqq 1
$$

This can always be achieved with a change of the time scale, and is used only for convenience in stating stability conditions.

2. The finite-difference scheme. We use a standard upwind-difference scheme to advance the approximate solution from time $t^{n}$ to $t^{n+1}$. We are given a suitable mesh, chosen by the rules in the next section, to represent the solution at time $t^{n}$. It is characterized by the meshpoints $x_{i}^{n}$ and the values of the approximate solution $U_{i}^{n}$ at those meshpoints. We interpret these points as determining a continuous piecewise linear approximation to $u\left(x, t^{n}\right)$. An estimate of the solution at time $t^{n+1}$ is calculated by

$$
\frac{\overline{U_{i}^{n+1}}-U_{i}^{n}}{\Delta t}+\frac{f^{+}\left(U_{i}^{n}\right)-f^{+}\left(U_{i-1}^{n}\right)}{h_{i}^{n}}+\frac{f^{-}\left(U_{i+1}^{n}\right)-f^{-}\left(U_{i}^{n}\right)}{h_{i+1}^{n}}=0
$$

for all $x_{i}^{n}$ (except the two endpoints of the interval), where $h_{i}^{n}=x_{i}^{n}-x_{i-1}^{n}$. We have decomposed $f$ into its increasing $\left(f^{+}\right)$and decreasing $\left(f^{-}\right)$parts. If $f$ is monotone increasing or decreasing then this method is an upwind difference method previously used by Engquist and Osher [11] on a uniform mesh.

The linear interpolant of the values $\overline{U_{i}^{n+1}}$ at the points $x_{i}^{n}$ is a function we call $\overline{u_{h}}$. The mesh selection algorithm of the next section, when applied to $\overline{u_{h}}$, gives us a new mesh $x_{i}^{n+1}$ and function values $U_{i}^{n+1}$ for the approximate solution of (C) at time $t^{n+1}$.

This process is repeated until $t^{n+1}=T$.

3. The mesh selection algorithm. This section describes our mesh selection algorithm. Although, in our implementation, the mesh at time $t^{n+1}$ is built from the mesh at time $t^{n}$, the method of approximation is general, and applies to any function without reference to a time-stepping procedure. It is presented here in its general form.

Our method of mesh selection is similar to well-known algorithms for adaptive linear approximation [3]. The mesh approximately equidistributes an estimate of the 
local error incurred by the finite-difference scheme, thus following methods used in static problems [4] and other evolution equations [7]. Because the solutions of (C) are stable in $L^{1}$, as described in the introduction, the mesh selection algorithm chooses to equidistribute the error in $L^{1}$. Our specific choice of the mesh will allow us to prove the stability results of $\S 4$, an important goal.

Let $u$ be any bounded function defined on $[a, b]$ that is constant outside of $[a, b]$, and let $\varepsilon$ be a small parameter. Choose the mesh according to the following algorithm.

Algorithm M. This algorithm chooses meshpoints at which to approximate an arbitrary function $u$ defined on $[a, b]$.

1. The meshpoints consist only of the points $a$ and $b$ and the centers of admissible intervals. Admissible intervals are defined by (2) and (3) below.

2. The interval $[a, b]$ is an admissible interval.

3. For any admissible interval $I$, let $3 I=\left\{x\left|\operatorname{dist}(x, I)=\inf _{y \in I}\right| x-y|<| I \mid\right\}$. If $|I| \geqq \varepsilon$ and

$$
|I| \int_{3 I}\left[\left|u_{x x}\right|+\left|f^{\prime \prime}(u)\right| u_{x}^{2}\right] d x \geqq \varepsilon,
$$

then the left and right halves of $I$ are admissible intervals. The above integral is finite if $u_{x x}$ is a finite measure; it is to be interpreted as $\infty$ otherwise. Note that $3 I$ is an open interval.

When this algorithm is used for our adaptive method, $\Delta t=\varepsilon / 4$, so that $\Delta t \leqq$ $\min _{i, n}\left(x_{i}^{n}-x_{i-1}^{n}\right)$.

A minimal interval is an admissible interval that contains no proper admissible subintervals. It is clear that the width of any given admissible interval is $2^{-k}(b-a)$ for some nonnegative $k$. Also, $|I| \geqq \varepsilon / 2$. It follows that the mesh is a subset of

$$
S_{\varepsilon}=\left\{a+m 2^{-k}(b-a) \mid m=0,1, \cdots, 2^{k} \text {, and }(b-a) 2^{-k} \geqq \varepsilon / 4>(b-a) 2^{-k-1}\right\} .
$$

Some lemmas about the structure of the mesh generated by this algorithm follow.

LEMMA 3.1. If $A$ and $B$ are two adjacent minimal intervals, then

$$
\frac{1}{2} \leqq \frac{|A|}{|B|} \leqq 2 \text {. }
$$

Proof. We let $|B|>2|A|$, and derive a contradiction. Since the width of any admissible interval is $(b-a) 2^{-k}$ for some $k \geqq 0,|B| \geqq 4|A|$. Consider now the admissible interval from which $A$ was derived by Step 3, and call it $C$. Since $C$ was divided into two admissible subintervals, the integral in Step 3 is greater than $\varepsilon$. However, $|B| \geqq 2|C|$ and $3 C \subset 3 B$, so that the corresponding integral for $B$ must also be greater than $\varepsilon$, a contradiction to the minimality of $B$.

Except for the two points $a$ and $b$, the set of meshpoints has the natural structure of a tree. The point $(a+b) / 2$ is the root of the tree. You can also think of the interval $[a, b]$ as the root of the tree. (Since there is a one-to-one correspondence between the meshpoints and the set of admissible intervals, we will describe the structure of the mesh equivalently in terms of intervals or meshpoints.) If an interval $I$ is divided into two admissible subintervals by Step 3, then these two intervals are the left and right children of $I$, and $I$ is their parent. A meshpoint with no children (the center of a minimal interval) is a leaf.

LEMMA 3.2. Every second meshpoint is a leaf.

Proof. The statement of the lemma is true after Step 2, and it is left invariant by Step 3. 
This implies the obvious result that there is a unique covering of $[a, b]$ with minimal intervals.

LEMMA 3.3. If the set of meshpoints is $\left\{x_{i}\right\}$ with $h_{i}=x_{i}-x_{i-1} \geqq 0$, then $\frac{1}{2} \leqq h_{i} / h_{i+1} \leqq 2$.

Proof. If $x_{i}$ is a leaf, then $h_{i}=h_{i+1}$. If $x_{i-1}$ and $x_{i+1}$ are leaves, then the result follows from Lemma 3.1.

The linear interpolant $u_{\varepsilon}$ of $u$ is defined by requiring that $u_{\varepsilon}\left(x_{i}\right)=u\left(x_{i}\right)$ for all $x_{i}$, and that $u_{\varepsilon}$ be a linear function between the meshpoints so that $u_{\varepsilon}$ is continuous on $[a, b]$. The function $u_{\varepsilon}$ has the following approximation properties (cf. [3]).

LEMMA 3.4.

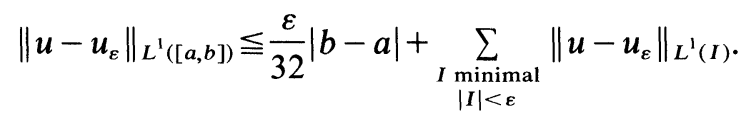

Proof. If $I=\left(x_{i-1}, x_{i+1}\right)$ is minimal and $|I| \geqq \varepsilon$ then Step 3 implies that $|I| \int_{I}\left|u_{x x}\right| d x \leqq \varepsilon$. The $L^{1}(I)$ error for linear interpolation at the points $x_{i-1}, x_{i}$, and $x_{i+1}$ is bounded by

$$
\frac{|I|^{2}}{32} \int_{I}\left|u_{x x}\right| d x \leqq \frac{|I|}{32} \varepsilon .
$$

Summing over all $I$ gives the first term in the expression. The second term contains all the intervals not yet considered.

There are two interesting cases when the second term is known to be small.

LEMMA 3.5. (a) If $u$ is a continuous, piecewise linear function such that $u_{x}$ is discontinuous only at the points in $S_{\varepsilon}$, then the second term in (3.2) is zero.

(b) If $u$ is in $B V(\mathbf{R})$ then the second term in (3.2) can be bounded by $\frac{1}{2}\|u\|_{B V(\mathbf{R})} \varepsilon$. Here we assume, without loss of generality, that

$$
u(x)=\lim _{h \rightarrow 0} \frac{1}{2 h} \int_{x-h}^{x+h} u(t) d t \text { for all } x .
$$

Proof. (a) Since $u$ is linear on each half of the minimal intervals $I$ with $|I|<\varepsilon$, $u_{\varepsilon}=u$ on the intervals.

(b) It is an exercise to show that $\left\|u_{\varepsilon}-u\right\|_{L^{1}(I)} \leqq \frac{1}{2} \varepsilon\|u\|_{B V(I)}$. Since $u$ is in $B V(\mathbf{R})$, we may add these individual bounds to obtain the lemma.

A few other stability properties will be used in the sequel.

LEMMA 3.6. $\left\|u_{\varepsilon}\right\|_{B V([a, b])} \leqq\|u\|_{B V([a, b])}$, and $\left\|u_{\varepsilon}\right\|_{L^{\infty}([a, b])} \leqq\|u\|_{L^{\infty}([a, b])}$.

Proof. It is clear that linear interpolation has these properties.

4. Stability properties. The numerical method presented here has several stability properties that mimic those for conservation laws. In brief, solutions of the numerical method satisfy a maximum principle, are total variation diminishing, and are stable in time. Although the numerical scheme is not conservative, one can bound the mass error for most problems.

Boundary effects will be analyzed in the following way. Outside of $[a, b]$ the mesh will be extended so that all the mesh intervals to the left of $a$ are of the same width as the mesh interval immediately to the right of $a$, denoted by $h_{a}$. A similar extension will be made to the right of $b$. We assume that the function $U_{i}^{n}$ is constant to the right and to the left of $[a, b]$. The mapping from $U_{i}^{n}$ to $U_{i}^{n+1}$ is now divided into three parts: the finite-difference scheme (2.1) transforms $U_{i}^{n}$ on the extended mesh to $\overline{U_{i}^{n+1}}$; the values at the points $a$ and $b$ are reset to their original values; and the remeshing procedure, applied to the mesh values in $[a, b]$, yields $U_{i}^{n+1}$. 
For the method to work properly, some criterion is needed to decide when a shock or other disturbance is getting too close to the boundary of the interval. Throughout, we will assume that the minimal intervals adjacent to the boundary points $a$ and $b$ have diameters greater than $\varepsilon$. This is a simple and effective criterion. If this criterion is in danger of being violated, the interval $[a, b]$ is to be enlarged.

We will follow the development of [9] for many of our proofs.

The following simple lemma will have important applications. then

LEMMA 4.1. If $g$ is $f^{+}, f^{-}$or $f^{t}$, and $u$ is the linear interpolant of the points $\left(x_{i}^{n}, U_{i}^{n}\right)$

$$
\left|\frac{g\left(U_{i+1}^{n}\right)-g\left(U_{i}^{n}\right)}{h_{i+1}^{n}}-\frac{g\left(U_{i}^{n}\right)-g\left(U_{i-1}^{n}\right)}{h_{i}^{n}}\right| \leqq \int_{x_{i-1}}^{x_{i+1}}\left[\left|u_{x x}\right|+\left|f^{\prime \prime}(u)\right| u_{x}^{2}\right] d x .
$$

Note that this is a multiple of the quantity that we use as a subdivision criterion.

Proof. Since $g$ has a bounded, piecewise continuous second derivative,

$$
g\left(U_{i+1}^{n}\right)=g\left(U_{i}^{n}\right)+h_{i+1}^{n} g^{\prime}\left(U_{i}^{n}\right) \frac{\left(U_{i+1}^{n}-U_{i}^{n}\right)}{h_{i+1}^{n}}+\int_{\left(x_{i}^{n}, x_{i+1}^{n}\right)}\left(x_{i+1}^{n}-x\right) g(u(x))_{x x} d x .
$$

Now, $g^{\prime}(u)$ is bounded by 1 , and for $x \in\left(x_{i}^{n}, x_{i+1}^{n}\right), g(u)_{x x}=g^{\prime \prime}(u) u_{x}^{2}$, since $u_{x x}=0$. Also, $\left|g^{\prime \prime}\right|$ is either $\left|f^{\prime \prime}\right|$ or 0 . The previous equation and a similar one for $g\left(U_{i-1}^{n}\right)$ can be rearranged and summed to yield

$$
\left|\frac{g\left(U_{i+1}^{n}\right)-g\left(U_{i}^{n}\right)}{h_{i+1}^{n}}-\frac{g\left(U_{i}^{n}\right)-g\left(U_{i-1}^{n}\right)}{h_{i}^{n}}\right| \leqq \int_{\left(x_{i-1}^{n}, x_{i+1}^{n}\right)}\left|u_{x x}\right|+\left|f^{\prime \prime}(u)\right| u_{x}^{2} d x .
$$

Here we have expressed the difference of the left and right derivatives of $u$ at $x_{i}^{n}$ as the integral of the second derivative "delta" measure.

The sharper bound

$$
\begin{aligned}
& \left|\frac{f^{+}\left(U_{i+1}^{n}\right)-f^{+}\left(U_{i}^{n}\right)}{h_{i+1}^{n}}-\frac{f^{+}\left(U_{i}^{n}\right)-f^{+}\left(U_{i-1}^{n}\right)}{h_{i}^{n}}\right| \\
& \quad+\left|\frac{f^{-}\left(U_{i+1}^{n}\right)-f^{-}\left(U_{i}^{n}\right)}{h_{i+1}^{n}}-\frac{f^{-}\left(U_{i}^{n}\right)-f^{-}\left(U_{i-1}^{n}\right)}{h_{i}^{n}}\right| \\
& \leqq \int_{\left(x_{i-1}^{n}, x_{i+1}^{n}\right)}\left[\left|u_{x x}\right|+\left|f^{\prime \prime}(u)\right| u_{x}^{2}\right] d x
\end{aligned}
$$

may be derived by noting that $f=f^{+}+f^{-}$, and if the first or second derivative of $f^{+}(u(x))$ is nonzero at $x$, then the first and second derivatives of $f^{-}(u(x))$ are zero there.

THEOREM 4.1. For all $n>0, \sup _{i} U_{i}^{n} \leqq \sup _{i} U_{i}^{0}$.

Proof. By (2.1),

$$
\overline{U_{i}^{n+1}}=U_{i}^{n}-\Delta t\left[\frac{f^{+}\left(U_{i}^{n}\right)-f^{+}\left(U_{i-1}^{n}\right)}{h_{i}^{n}}+\frac{f^{-}\left(U_{i+1}^{n}\right)-f^{-}\left(U_{i}^{n}\right)}{h_{i+1}^{n}}\right] .
$$

Since $f^{+}$is increasing, $f^{-}$is decreasing, $\left\|f^{t}\right\|_{L i p} \leqq 1$, and $\Delta t / h_{i}^{n} \leqq 1$, the preceding equation yields

$$
\begin{aligned}
\overline{U_{i}^{n+1}} & \leqq U_{i}^{n}-\Delta t\left[\frac{f^{+}\left(U_{i}^{n}\right)-f^{+}\left(U_{i-1}^{n} \vee U_{i}^{n} \vee U_{i+1}^{n}\right)}{h_{i}^{n}}+\frac{f^{-}\left(U_{i-1}^{n} \vee U_{i}^{n} \vee U_{i+1}^{n}\right)-f^{-}\left(U_{i}^{n}\right)}{h_{i+1}^{n}}\right] \\
& \leqq U_{i}^{n}+f^{t}\left(U_{i-1}^{n} \vee U_{i}^{n} \vee U_{i+1}^{n}\right)-f^{t}\left(U_{i}^{n}\right) \\
& \leqq U_{i-1}^{n} \vee U_{i}^{n} \vee U_{i+1}^{n} .
\end{aligned}
$$


Therefore, the finite-difference scheme satisfies a maximum principle. Resetting the values of $U_{i}^{n}$ at $a$ and $b$ does not violate a maximum principle. Lemma 3.6 states that the subsequent transformation to $U^{n+1}$ satisfies a maximum principle.

THEOREM 4.2. For all $n>0,\left\|U^{n}\right\|_{B V(R)} \leqq\left\|U^{0}\right\|_{B V(R)}$.

Proof. If we let $\delta f_{i}^{+}=f^{+}\left(U_{i}^{n}\right)-f^{+}\left(U_{i-1}^{n}\right)$, then

$$
\overline{U_{i}^{n+1}}-\overline{U_{i-1}^{n+1}}=U_{i}^{n}-U_{i-1}^{n}-\Delta t\left[\frac{\delta f_{i}^{+}}{h_{i}^{n}}-\frac{\delta f_{i-1}^{+}}{h_{i-1}^{n}}+\frac{\delta f_{i+1}^{-}}{h_{i+1}^{n}}-\frac{\delta f_{i}^{-}}{h_{i}^{n}}\right] .
$$

Because $f^{+}$and $f^{-}$are monotone, $f^{t}=f^{+}-f^{-},\left\|f^{t}\right\|_{L i p} \leqq 1$ and $\Delta t / h_{i}^{n} \leqq 1$, we can take absolute values, and sum:

$$
\begin{aligned}
\sum_{i}\left|\overline{U_{i}^{n+1}}-\overline{U_{i-1}^{n+1}}\right| & \leqq \sum_{i}\left|U_{i}^{n}-U_{i-1}^{n}-\frac{\Delta t}{h_{i}^{n}}\left[f^{t}\left(U_{i}^{n}\right)-f^{t}\left(U_{i-1}^{n}\right)\right]\right|+\Delta t \sum_{i}\left[\left|\frac{\delta f_{i-1}^{+}}{h_{i-1}^{n}}\right|+\left|\frac{\delta f_{i+1}^{-}}{h_{i+1}^{n}}\right|\right] \\
& =\sum_{i}\left|U_{i}^{n}-U_{i-1}^{n}\right| .
\end{aligned}
$$

Thus the mapping $U^{n} \rightarrow \overline{U^{n+1}}$ is total variation diminishing. Resetting the values at the endpoints does not increase the total variation. Since the remeshing process is variation diminishing, by Lemma 3.6, the theorem is proved.

THEOREM 4.3. $\left\|U^{n+1}-U^{n}\right\|_{L^{1}([a, b])} \leqq\left(\frac{3}{2}\left\|U^{0}\right\|_{B V(R)}+(b-a) / 8\right) \Delta t+2 \Delta t \varepsilon$.

Proof. First, since the mesh is graded,

$$
\begin{aligned}
\left\|\overline{U^{n+1}}-U^{n}\right\|_{L^{1}([a, b])} \leqq \Delta t \sum_{i}\left|\overline{U_{i}^{n+1}}-U_{i}^{n}\right| \frac{\left(h_{i}^{n}+h_{i+1}^{n}\right)}{2} \\
\leqq \frac{\Delta t}{2} \sum_{i}\left[\left|f^{+}\left(U_{i}^{n}\right)-f^{+}\left(U_{i-1}^{n}\right)\right|\left(1+\frac{h_{i+1}^{n}}{h_{i}^{n}}\right)\right. \\
\left.\quad \quad+\left|f^{-}\left(U_{i+1}^{n}\right)-f^{-}\left(U_{i}^{n}\right)\right|\left(1+\frac{h_{i}^{n}}{h_{i+1}^{n}}\right)\right] \\
\leqq \frac{3 \Delta t}{2} \sum_{i}\left|f^{t}\left(U_{i}^{n}\right)-f^{t}\left(U_{i-1}^{n}\right)\right| \\
\leqq \frac{3 \Delta t}{2}\left\|U^{0}\right\|_{B V(\mathbf{R}) .}
\end{aligned}
$$

When the boundary values are restored, we commit an error at the left endpoint of at most $\Delta t \mid U_{1}^{n}-U_{0}^{n}$. Under the assumption that the width of the minimal interval adjacent to $a$ is bigger than $\varepsilon$, Step 3 of the mesh construction allows us to bound the error by $\Delta t \varepsilon$.

Finally, we may apply Lemmas 3.4 and 3.5 to yield

$$
\left\|U^{n+1}-\overline{U^{n+1}}\right\|_{L^{1}([a, b])} \leqq(b-a) \Delta t / 8 \text {. }
$$

Because a consistent but nonconservative finite-difference operator is used for the time-stepping, there will, in general, be some mass balance error. The following theorem bounds the mass error with an estimate that involves the number of minimal intervals of width greater than $\varepsilon$. It may be shown that, for functions whose first derivative is of bounded variation on an interval $I$, the mesh selection algorithm generates on the order of $C \varepsilon^{-1 / 2}\left\|u^{\prime}\right\|_{B V(I)}$ meshpoints in $I$ (see, for example, de Boor [3]). Consequently, the number of large minimal intervals may be seen as an estimate of the size of the second derivative of the function. Similarly, it may also be shown that, if the initial 
data for $(C)$ is piecewise smooth and of bounded variation, there are fewer than $C \varepsilon^{-1 / 2}$ meshpoints in the smooth regions. We have observed in all computational tests that as the integration in time progresses this bound on the number of large minimal intervals continues to hold. We have therefore applied this hypothesis in a number of theorems. When applied to the following mass error bound, it implies a mass balance error of $O\left(\varepsilon^{1 / 2}\right)$, the expected convergence rate of the method, and a rate which matches the observed rates in $\$ 7$ (see especially Example 5).

Theorem 4.4. Assume $[a, b]=\bigcup_{j} S_{j} \cup \cup_{j} R_{j}$ where $m-1$ disjoint regions $R_{j}$ are made up of minimal intervals of width less that $\varepsilon$, and are surrounded by $m$ regions $S_{j}$ that are covered by minimal intervals whose width is greater than $\varepsilon$. Assume that there are no more than $k$ minimal intervals in $\bigcup_{j} S_{j}$. Then

$$
\left|\int_{\mathbf{R}} \overline{U^{n+1}}-U^{n} d x-\Delta t(f(u(a))-f(u(b)))\right| \leqq \Delta t\left(2 k \varepsilon+\left(2 m \varepsilon\left\|U^{n}\right\|_{B V(\mathbf{R})}\right)^{1 / 2}\right) .
$$

Proof. Using the notation of Theorem 4.2, we have

$$
\begin{aligned}
& \frac{1}{\Delta t} \int_{\mathbf{R}} \overline{U^{n+1}(x)}-U^{n}(x) d x \\
& \quad=-\sum_{i}\left(\frac{\delta f_{i}^{+}}{h_{i}^{n}}+\frac{\delta f_{i+1}^{-}}{h_{i+1}^{n}}\right)\left(\frac{h_{i}^{n}+h_{i+1}^{n}}{2}\right) \\
& \quad=\frac{1}{2}\left[f^{+}(u(a))+f^{-}(u(a))-\left(f^{+}(u(b))+f(u(b))\right)\right]-\frac{1}{2} \sum_{i}\left(\frac{\delta f_{i}^{+}}{h_{i}^{n}} h_{i+1}^{n}+\frac{\delta f_{i+1}^{-}}{h_{i+1}^{n}} h_{i}^{n}\right) \\
& \quad=\frac{1}{2}(f(u(a))-f(u(b)))-\frac{1}{2} \sum_{i} \frac{\delta f_{i}^{+}}{h_{i}^{n}} h_{i+1}^{n}-\frac{1}{2} \sum_{i} \frac{\delta f_{i+1}^{-}}{h_{i+1}^{n}} h_{i}^{n} .
\end{aligned}
$$

We will show that the first sum minus $\frac{1}{2}\left(f^{+}(u(a))-f^{+}(u(b))\right)$ can be bounded as in the statement of the theorem.

Pick a particular $S_{j}$ with left and right endpoints $x_{l}$ and $x_{r}$ and consider the sum

$$
\sum_{x_{i}^{n}=x_{l}}^{x_{r}} \frac{\delta f_{i}^{+}}{h_{i}^{n}} h_{i+1}^{n} .
$$

Since every minimal interval contained in $S_{j}$ is more than $\varepsilon$ wide, Step 3 of Algorithm $M$ and Lemma 3.5 show that (4.4) is equal to

$$
\sum_{x_{i}^{n}=x_{l}}^{x_{r}} \frac{\delta f_{i+1}^{+}}{h_{i+1}^{n}} h_{i+1}^{n}+M=\sum_{x_{l+1}}^{x_{r+1}} \frac{\delta f_{i}^{+}}{h_{i}^{n}} h_{i}^{n}+M,
$$

where $|M|$ is less than $\varepsilon(r-l+1)$.

Any interval $R_{j}$ consists of minimal intervals of width less than $\varepsilon$. Perforce, all the intervals must have the same width. Thus, if $x_{l}$ and $x_{r}$ are the left and right boundaries of $R_{j}$,

$$
\sum_{x_{i}^{n}=x_{t+1}}^{x_{r-1}} \frac{\delta f_{i}^{+}}{h_{i}^{n}} h_{i+1}^{n}=\sum_{x_{i}^{n}=x_{l+1}}^{x_{r-1}} \frac{\delta f_{i}^{+}}{h_{i}^{n}} h_{i}^{n} .
$$

Matching these results gives

$$
\sum_{i} \frac{\delta f_{i}^{+}}{h_{i}^{n}} h_{i+1}^{n}=\sum_{i} \frac{\delta f_{i}^{+}}{h_{i}^{n}} h_{i}^{n}+M+\sum_{s_{i}=\left(x_{l}, x_{r}\right)}\left(\delta f_{r+1}^{+}-\delta f_{l}^{+}\right) .
$$

Here, $|M| \leqq 2 k \varepsilon$, since $\cup S_{j}$ is covered by $k$ minimal intervals. The remark following 
Lemma 4.1 implies that the same bound holds when the terms incorporating $f^{-}$are also included. The first sum on the right of (4.5) is equal to $f^{+}(u(b))-f^{+}(u(a))$.

For the interval $S_{j}$, assume $\left|\delta f_{r+1}^{+} / h_{r+1}^{n}-\delta f_{l}^{+} / h_{l}^{n}\right|=K_{j}$, for some positive $K_{j}$. Then, without loss of generality, we may assume that $\delta f_{r+1}^{+} / h_{r+1}^{n} \geqq K_{j} / 2$. Lemma 4.1 and Step 3 of the mesh construction imply that $\left|\delta f_{i+1}^{+} / h_{i+1}^{n}-\delta f_{i}^{+} / h_{i}^{n}\right| \leqq 1$ if $x_{i}^{n} \in S_{j}$. Also, $h_{i}^{n} \geqq \varepsilon / 2$ for $x_{i}^{n} \in S_{j}$. Therefore,

$$
\left\|f^{+}\left(U^{n}\right)\right\|_{B V\left(S_{j}\right)} \geqq \frac{\varepsilon}{2} \sum_{i=0}^{K_{j} / 2}\left(\frac{K_{j}}{2}-i\right) \geqq \frac{\varepsilon K_{j}^{2}}{8} .
$$

Thus, the last sum in (4.5) is bounded by

$$
\frac{\varepsilon}{2} \sum_{j} K_{j} \leqq(2 \varepsilon m)^{1 / 2}\left(\varepsilon \sum_{j} K_{j}^{2} / 8\right)^{1 / 2} \leqq\left(2 \varepsilon m\left\|f^{+}\left(U^{n}\right)\right\|_{B V(\mathbf{R})}\right)^{1 / 2} .
$$

Since $\left\|\left.f^{+}\left(U^{n}\right)\right|_{B V(\mathbf{R})}+\right\| f^{-}\left(U^{n}\right)\left\|_{B V(\mathbf{R})} \leqq\right\| U^{n} \|_{B V(\mathbf{R})}$, the theorem is proved.

Unfortunately, there is no a priori bound on the number of meshpoints in a mesh generated by Algorithm $M$ for a general function in $B V(\mathbf{R})$; because we have a priori bounds only on the variation of $u_{\varepsilon}, \varepsilon^{-1}$ meshpoints may be needed to approximate it. The numbers $k$ and $m$ can be calculated during the course of the algorithm in a negligible amount of computer time, however, and (4.3) may be used as an a posteriori bound.

Theorem 4.4 does not consider the mass error generated by the adjustment of the function values at the points $a$ and $b$. Theorem 4.3 shows that this error is $O(\Delta t)$ if the minimal intervals next to $a$ and $b$ have width greater than $\varepsilon$.

Estimate (4.3) does not include the mass error caused by the remeshing process. Such an error occurs when admissible intervals in the mesh at time $t^{n}$ are no longer needed, and do not appear in the mesh at time $t^{n+1}$. Lemma 3.4 is sharp for any given time step; the mass error for a time step of size $\Delta t$ can be comparable to $\Delta t$. Using this bound over the interval $[0, T]$ gives an estimate of an $O(1)$ mass error, a totally unacceptable result.

Computational experience with piecewise smooth solutions of the differential equation has shown that over the interval $[0, T]$ there is a constant $C$ such that fewer than $C 2^{k}$ intervals of width $2^{-k}(b-a)$ are removed from the mesh in $[0, T]$. That is, a minimal interval is not subsumed into a larger minimal interval for a period of time proportional to its width. If this property holds, an argument similar to that of Lemma 3.4 shows that the mass error due to mesh changes on $[0, T]$ is bounded by $C \Delta t \log \left(\Delta t^{-1}\right)$ for some $C$. Combining our previous hypotheses, we have the following theorem.

TheOREM 4.5. For an interval $[0, T]$, assume that there are constants $C_{1}, C_{2}$, and $C_{3}$ such that for any $\varepsilon>0$ :

(a) there are no more than $C_{1} \varepsilon^{-1 / 2}$ minimal intervais of width bigger than $\varepsilon$ for any $t^{n}$ in $[0, T]$

(b) there are never more than $C_{2}$ disjoint regions covered by minimal intervals of width less than $\varepsilon$; and

(c) at most $C_{3} 2^{k}$ admissible intervals of width $(b-a) 2^{-k}$ are removed from the mesh in $[0, T]$.

Then there exists a constant $C$, depending on $C_{1}, C_{2}, C_{3}$, and $\|u\|_{B V(\mathbf{R})}$, such that the mass error of the numerical approximation (2.1) can be bounded by $C \varepsilon^{1 / 2}$.

The conditions (a), (b), and (c) can easily be checked a posteriori; they have been observed to hold for all the experiments in $\S 7$. 
5. Convergence for linear problems. In this section we prove that solutions of the linear problem

$$
\begin{aligned}
& u_{t}+\alpha u_{x}=0, \quad x \in \mathbf{R}, t>0, \\
& u(x, 0)=u_{0}(x), \quad x \in \mathbf{R}
\end{aligned}
$$

are approximated well by variants of our adaptive mesh algorithm. If the initial data is in $B V(\mathbf{R})$, a posteriori bounds will be given on the error, and if the initial data is slightly smoother, with $d u_{0} / d x \in B V(\mathbf{R})$, a priori bounds are available. Formal justification of the mesh selection criteria used in Algorithm $M$ is also given.

Consider first when $u_{0} \in B V(\mathbf{R})$. Our algorithm is as follows:

1. Pick $\varepsilon>0$, and let $u_{0}^{\varepsilon}=\psi_{\varepsilon} * u_{0}$, where $\psi_{\varepsilon}(x)$ is $\varepsilon^{-1 / 2}$ when $|x| \leqq \varepsilon^{1 / 2} / 2$ and 0 otherwise.

2. Using $u_{0}^{\varepsilon}$ as the initial data, follow the algorithm in $\S 2$ to find $u^{\varepsilon}$. This will be our approximation to $u$.

It is easily shown that $\left\|d u_{0}^{\varepsilon} / d x\right\|_{B V(\mathbf{R})} \leqq \varepsilon^{-1 / 2}\left\|u_{0}\right\|_{B V(\mathbf{R})}$, and $\left\|u_{0}-u_{0}^{\varepsilon}\right\|_{L^{1}(\mathbf{R})} \leqq$ $\varepsilon^{1 / 2}\left\|u_{0}\right\|_{B V(\mathbf{R})}$.

The following theorem applies to such problems. See the discussion following Theorem 4.3 regarding the suitability of the hypotheses of the following theorem.

THEOREM 5.1. If, at each timestep, there are never more than $C_{1} \varepsilon^{-1 / 2}$ minimal intervals of width greater than $\varepsilon$; and if the total $L^{1}([a, b])$ error due to mesh changes is $O\left(\varepsilon^{1 / 2}\right)$, then

$$
\left\|u(t)-u^{\varepsilon}(t)\right\|_{L^{1}([a, b])} \leqq C_{2}(t+1) \varepsilon^{1 / 2}\left(\left\|u_{0}\right\|_{B V(\mathbf{R})}+1\right) .
$$

Furthermore, the computational complexity of the scheme is $O\left(\varepsilon^{-3 / 2}\right)$.

Theorem 5.1 gives a posteriori estimates of the error and computational complexity of the scheme. Our computational experience suggests that the conditions of the theorem are always satisfied. We suspect that the structure of solutions of the conservation laws ensures this. The computational bound is better than for standard monotone methods; for these mehods the error is $O\left(\Delta t^{1 / 2}\right)$ but the complexity is $O\left(\Delta t^{-2}\right)$.

The proof of this theorem relies on a number of lemmas.

LEMMA 5.1. If $u_{x} \in B V(\mathbf{R})$ and $v$ is the linear interpolant of $u$ on some mesh, $\left\{x_{i}\right\}$, then $\left\|v_{x}\right\|_{B V(\mathbf{R})} \leqq\left\|u_{x}\right\|_{B V(\mathbf{R})}$.

Proof. Since

$$
v_{x}(x)=\frac{1}{x_{i}-x_{i-1}} \int_{x_{i-1}}^{x_{i}} u_{x}(t) d t \quad \text { for } x \in\left(x_{i-1}, x_{i}\right)
$$

the result follows immediately.

Lemma 5.2. If $U^{n}$ is generated by Algorithm $\mathrm{M}$ and (2.1), then

$$
\left\|U_{x}^{n}\right\|_{B V(\mathbf{R})} \leqq\left\|U_{x}^{0}\right\|_{B V(\mathbf{R})} .
$$

Proof. Since (5.1) is linear, $V_{i}^{n}=\left(U_{i}^{n}-U_{i-1}^{n}\right) / h_{i}$ satisfies the same difference equation as $U_{i}^{n}$. Hence $\left\|V^{n+1}\right\|_{B V(\mathbf{R})} \leqq\left\|V^{n}\right\|_{B V(\mathbf{R})}$. By the previous lemma, $\left\|V^{n+1}\right\|_{B V(\mathbf{R})} \leqq$ $\left\|V^{n+1}\right\|_{B V(\mathbf{R})}$. The result then follows by induction.

Our analysis views upwind finite-difference schemes for conservation laws in a way that is very close to that used by Courant, Isaacson, and Rees in [5]. In particular, if $U_{i}^{n}$ and $U_{i}^{n+1}$ are interpreted as piecewise linear functions, then the equation $(2.1)$ defining $\overline{U_{i}^{n+1}}$ is equivalent to the following algorithm.

To obtain $\overline{U_{i}^{n+1}}$, shift $U_{i}^{n}$ to the right by $\alpha \Delta t$ (if $\alpha$ is positive) and interpolate the shifted function at the points $x_{i}^{n}$. The calculation is illustrated in Fig. 1 . 


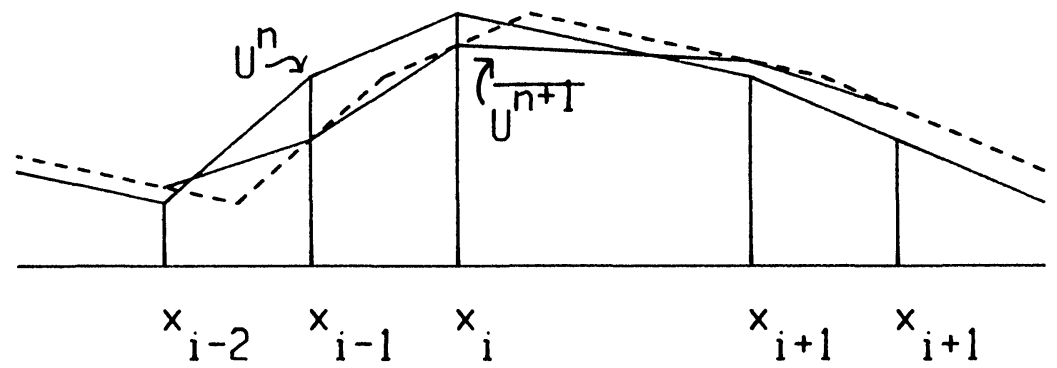

FIG. 1. The error caused by timestepping. The dashed line represents $\sigma\left(U^{n}\right)$.

Note that error occurs only in the interpolation process; there is no error in the values of the $U_{i}^{n+1}$ themselves if the values of the $U_{i}^{n}$ are exact.

Our interpretation is somewhat different from the piecewise constant upwinddifference scheme, in that our scheme averages the first spatial derivative of the solution at the advanced time level to get the new approximation, while the piecewise constant method averages the solution itself. Whereas the local error of the piecewise constant method depends on the variation of the solution, it will be shown that the local error in our scheme depends on the variation of the solution's first derivative. (Piecewise linear approximations for conservation laws were previously used by van Leer [23]. Leveque [18] exploits the idea of difference schemes as projections of an exact solution onto a finite-dimensional vector space.)

Proof of Theorem 5.1. We first define $\sigma(v)(x)=v(x-\alpha \Delta t)$. If $e_{n}=$ $\left\|u(n \Delta t)-U^{n}\right\|_{L^{1}([a, b])}$ then

$$
\begin{aligned}
e_{n} & \leqq\left\|u(n \Delta t)-\sigma\left(U^{n-1}\right)\right\|_{L^{1}([a, b])}+\left\|\sigma\left(U^{n-1}\right)-U^{n}\right\|_{L^{1}([a, b])} \\
& =\left\|\sigma(u((n-1) \Delta t))-\sigma\left(U^{n-1}\right)\right\|_{L^{1}([a, b])}+\left\|\sigma\left(U^{n-1}\right)-U^{n}\right\|_{L^{1}([a, b])} \\
& =e_{n-1}+\delta_{n},
\end{aligned}
$$

where $\delta_{n}$ is the local error $\left\|\sigma\left(U^{n-1}\right)-U^{n}\right\|_{L^{1}([a, b])}$. We define $\delta U_{i}^{n}=\left(U_{i}^{n}-U_{i-1}^{n}\right) / h_{i}^{n}$. Now, from Fig. 1,

$$
\begin{aligned}
\left\|\overline{U^{n+1}}-\sigma\left(U^{n}\right)\right\|_{L^{1}([a, b])} & \sum_{x_{i}^{n}} \alpha \Delta t h_{i}^{n}\left(1-\frac{\alpha \Delta t}{h_{i}^{n}}\right)\left|\delta U_{i}^{n}-\delta U_{i-1}^{n}\right| \\
\leqq & 2 \alpha \Delta t^{2} \sum_{h_{i}^{n}<2 \Delta t}\left|\delta U_{i}^{n}-\delta U_{i-1}^{n}\right| \\
& +\alpha \Delta t \sum_{h_{i}^{n} \geqq 2 \Delta t} h_{i}^{n}\left|\delta U_{i}^{n}-\delta U_{i-1}^{n}\right| .
\end{aligned}
$$

By using Lemmas 5.1 and 5.2, one can bound the first term by

$$
C \Delta t \varepsilon\left\|U_{x}^{n}\right\|_{B V(\mathbf{R})} \leqq C \varepsilon^{1 / 2}\left\|u_{0}\right\|_{B V(\mathbf{r})} \Delta t .
$$

Because of Step 3 of Algorithm M, and our assumption about the number of intervals of width greater than $\varepsilon$, the second term can be bounded by

$$
\alpha \Delta t \varepsilon \times C \varepsilon^{-1 / 2}=C \varepsilon^{1 / 2} \Delta t .
$$

Thus $\left\|\overline{U^{n+1}}-\sigma\left(U^{n}\right)\right\|_{L^{1}([a, b])} \leqq C \Delta t \varepsilon^{1 / 2}\left(\left\|u_{0}\right\|_{B V(\mathbf{R})}+1\right)$.

Summing this expression over $n \leqq t / \Delta t$ gives one term of (5.2). We have assumed that

$$
\sum_{n}\left\|\overline{U^{n+1}}-U^{n+1}\right\|_{L^{1}([a, b])} \leqq C \varepsilon^{1 / 2}
$$


As stated previously, $\left\|u_{0}-u_{0}^{\varepsilon}\right\| \leqq \varepsilon^{1 / 2}\left\|u_{0}\right\|_{B V(\mathbf{R})}$. Lemmas 3.4 and 3.5 show that

$$
\left\|U^{0}-u_{0}^{\varepsilon}\right\|_{L^{1}([a, b])} \leqq \frac{\varepsilon(b-a)}{32}+\frac{1}{2} \varepsilon\left\|u_{0}\right\|_{B V(\mathbf{R})} .
$$

The error bound is proved.

The complexity of the scheme is $O\left(\Delta t^{-1} N\right)$ where $N$ is the maximum number of meshpoints in any mesh $\left\{x_{i}^{n}\right\}$, since the amount of work is linear in the number of meshpoints. By hypothesis, there are fewer than $C \varepsilon^{-1 / 2}$ minimal intervals of width greater than $\varepsilon$. Furthermore, since $\int_{3 I}\left|u_{x x}\right| d x>1$ if $I<\varepsilon$, there must be fewer than $3\left\|U_{x}^{n}\right\|_{B V(\mathbf{R})} \leqq 3 \varepsilon^{-1 / 2}\left\|u_{0}\right\|_{B V(\mathbf{R})}$ minimal intervals of width less than $\varepsilon$. Because every second meshpoint is the center of a minimal interval, the theorem is proved.

We now consider the case when $u$ is smoother. Let $L^{1,2}(\mathbf{R})=$ $\left\{u \in L^{1}(\mathbf{R}) \cap B V(\mathbf{R}) \mid u_{x} \in B V(\mathbf{R})\right\}$. Although functions in $L^{1,2}(\mathbf{R})$ have first derivatives of bounded variation, they need not be $C^{1}$; in fact, their first derivatives may be discontinuous on a countable, dense set.

We define here a modification of Algorithm $M$ for functions in $L^{1,2}(\mathbf{R})$.

Algorithm M'.

1. The points $a$ and $b$ are meshpoints. The center of every admissible interval (to be defined below) is a meshpoint.

2. The interval $[a, b]$ is an admissible interval.

3. If $I$ is an admissible interval, $|I| \geqq 4 \Delta t, 3 I=\{x|\operatorname{dist}(x, I)<| I \mid\}$, and

$$
|I| \int_{3 I}\left|u_{x x}\right| d x>\frac{\left\|u^{\prime}\right\|_{B V(\mathbf{R})}}{(b-a)}(\Delta t)^{2},
$$

then the left and right halves of $I$ are admissible intervals. As before, $u_{x x}$ is to be interpreted as a measure.

This new mesh algorithm makes the smallest admissible intervals the same size as the "average" interval, which occurs where $u_{x x}$ is bounded. Since $u \in L^{1,2}(\mathbf{R})$, the very small spatial and temporal mesh increments of the previous method are not needed. The following adaptive algorithm is therefore a true "smooth solution" algorithm.

Our new algorithm is as follows:

(a) Given $u_{0}(x)$, find $x_{i}^{0}$ using Algorithm $\mathrm{M}^{\prime}$ and choose $U_{i}^{0}$ using piecewise linear interpolation of $u_{0}$.

(b) For each timestep: Given meshpoints $\left\{x_{i}^{n}\right\}$ and function values $\left\{U_{i}^{n}\right\}$ defined at those meshpoints, calculate $\overline{U_{i}^{n+1}}$ using (2.1). (Equation (2.1) reduces to the upwind differencing scheme in this case.) Interpreting $\overline{U_{i}^{n}}$ as a continuous, piecewise linear function, use algorithm $\mathrm{M}^{\prime}$ to find a new mesh $\left\{x_{i}^{n+1}\right\}$ for $\overline{U_{i}^{n+1}}$ and define $U_{i}^{n+1}$ on that mesh by linear interpolation of $\overline{U_{i}^{n+1}}$.

The following result holds.

THEOREM 5.2. Let $u_{0} \in L^{1,2}(\mathbf{R})$ and $u(x, t)$ be the solution of (5.1). If $n \Delta t=T$, and $U^{n}$ is the solution of the adaptive mesh algorithm above, then

$$
\left\|u(T)-U^{n}\right\|_{L^{1}([a, b])} \leqq 3(T+1) \Delta t\left\|u_{0}^{\prime}\right\|_{B V(\mathbf{R})} .
$$

Proof. As in Theorem 5.1.

$$
\left\|\overline{U^{n+1}}-\sigma\left(U^{n}\right)\right\|_{L^{1}([a, b])} \leqq 2 \alpha \Delta t^{2} \sum_{h_{i}^{n}<2 \Delta t}\left|\delta U_{i}^{n}-\delta U_{i-1}^{n}\right|+\alpha \Delta t \sum_{h_{i}^{n} \geqq 2 \Delta t} h_{i}^{n}\left|\delta U_{i}^{n}-\delta U_{i-1}^{n}\right| .
$$

The first term is bounded by $2 \alpha \Delta t^{2}\left\|u_{0}^{\prime}\right\|_{B V(\mathbf{R})}$ because of Lemmas 5.1 and 5.2. By using 
Step 3 of Algorithm $\mathbf{M}^{\prime}$, the second term can be bounded by

$$
\begin{aligned}
\alpha \Delta t\left(\sum_{h_{i}^{n} \geqq 2 \Delta t} h_{i}^{n}\right)^{1 / 2}\left(\sum_{h_{i}^{n} \geqq 2 \Delta t} h_{i}^{n}\left|\delta U_{i}^{n}-\delta U_{i-1}^{n}\right|^{2}\right)^{1 / 2} & \leqq \alpha \Delta t(b-a)^{1 / 2} \Delta t \frac{\left\|U_{x}^{n}\right\|_{B V(\mathbf{R})}}{(b-a)^{1 / 2}} \\
& \leqq \alpha \Delta t^{2}\left\|u_{0}^{\prime}\right\|_{B V(\mathbf{R})} .
\end{aligned}
$$

Thus $\left\|\overline{U^{n+1}}-\sigma\left(U^{n}\right)\right\|_{L^{1}([a, b])} \leqq 3 \alpha \Delta t^{2}\left\|u_{0}^{\prime}\right\|_{B V(\mathbf{R})}$.

Finally, let $S$ be the set of all minimal intervals in the mesh at time $t^{n+1}$ that are not minimal at time $t^{n}$. Then, because of Lemma 3.4 and Step 3 of Algorithm $\mathrm{M}^{\prime}$, the expression for the error in the trapezoid rule yields

$$
\begin{aligned}
\left\|\overline{U^{n+1}}-U^{n+1}\right\|_{L^{1}([a, b])} & \leqq \sum_{\substack{I \in S \\
x_{i}^{n} \in I}} \frac{|I|^{2}}{32} \mid \delta \overline{U_{i}^{n}}-\delta \overline{U_{i-1}^{n} \mid} \\
& \leqq \frac{\Delta t^{2}\left\|u_{0}^{\prime}\right\|_{B V(\mathbf{R})}}{32(b-a)} \sum_{I \in S}|I| \leqq \frac{\Delta t^{2}}{32}\left\|u_{0}^{\prime}\right\|_{B V(\mathbf{R}) .}
\end{aligned}
$$

Thus, $\delta_{n} \leqq 3 \Delta t^{2}\left\|u_{0}^{\prime}\right\|_{B V(\mathbf{R})}$. After calculating the error caused by the approximation of $u_{0}$, the theorem follows by induction.

The same analysis can be used formally for nonlinear problems: estimate the difference, now nonzero, between the values $\overline{U_{i}^{n+1}}$ and $S_{\Delta t}\left(U^{n}\right)\left(x_{i}^{n}\right),\left(S_{\Delta t}\right.$ advances the solution of $(C)$ by time $\Delta t)$ and then separately estimate the interpolation error as in Fig. 1. The error in $U_{i}^{n+1}$ is bounded formally by

$$
\Delta t \int_{x_{i-1}^{n}}^{x_{i+1}^{n}}\left|f\left(U^{n}\left(x, t^{n}\right)\right)_{x x}\right| d x+O\left(\Delta t^{2}\right) .
$$

The $L^{1}$ error on $\left(x_{i-1}^{n}, x_{i+1}^{n}\right)$ is therefore bounded by $\left(h_{i}^{n}+h_{i+1}^{n}\right) / 2$ times $(5.4)$, bounded in turn by $C \Delta t$ times the integral in (3.1).

Thus, if $\left\|U_{x}^{n+1}\right\|_{B V(I)} \leqq C\left\|U_{x}^{n}\right\|_{B V(3 I)}$ for each minimal interval $I$, the error caused by the nonlinearity is of the same order as the error caused by the dissipation in the difference scheme. Thus, our mesh selection criterion achieves a balance between errors caused by nonlinearity and dissipation.

6. Implementation details. Our scheme was implemented using the programming language Pascal. The resulting programs were run on a VAX 11/780 with a floating point accelerator under the VMS 2.5 operating system and Pascal 1.2 compiler, and under the Berkeley Unix 4.1 operating system with its Pascal compiler. In this section, we describe the algorithms and data structures used in our implementation. We show that the integrals in (3.1) can be evaluated exactly, and hence that the stability results of the previous section hold without a separate theory describing the effects of numerical quadrature. We also estimate the computational complexity of the scheme.

The following steps advance the approximate solution from one timestep to the next. First, the finite-difference formula advances $u_{h}$ from $t^{n}$ to $t^{n+1}$ on the mesh $\left\{x_{i}^{n}\right\}$. Secondly, the integrals in (3.1) are calculated for the mesh $\left\{x_{i}^{n}\right\}$. In our implementation, the integrals are first calculated over the (open) interval $I$ instead of $3 I$, and the integral over $3 I$ is constructed when it is needed. Finally, the mesh $\left\{x_{i}^{n+1}\right\}$ is constructed. To do this, the union of the meshes $\left\{x_{i}^{n}\right\}$ and $\left\{x_{i}^{n+1}\right\}$ is built up by adding the appropriate meshpoints to $\left\{x_{i}^{n}\right\}$. The values of the integrals (3.1) are derived for the new meshpoints as they are introduced. A second pass is made to remove points in $\left\{x_{i}^{n}\right\}$ that are not needed in $\left\{x_{i}^{n+1}\right\}$. 
Because the mesh is defined recursively by subdividing admissible intervals into two subintervals at each step, the mesh is naturally organized as a (threaded) binary tree (see Knuth [15]). Each admissible interval (or, equivalently, the meshpoint at its center) is a node in the tree; the interval $[a, b]$ is the root of the tree. If an admissible interval is subdivided into two admissible subintervals, then these subintervals are the left and right children of the interval. A node without children is a leaf, and corresponds to a minimal interval. Nodes that are not leaves are interior nodes. The parent of an interval is the admissible interval from which it was derived.

We first describe the information stored in a node corresponding to a meshpoint $x_{i}^{n}$ at the center of an interval $I=\left(x_{l}, x_{r}\right)$. This information consists mainly of numeric variables and pointer variables, each of which either points to the beginning of a block of computer store allocated to a node, or is nil. A pointer has the value nil when it points to "nothing", i.e. it does not point to the memory location of a node. For example, the pointer "parent" points to the information for the parent node of $x_{i}^{n}$. The numeric variables contain such information as the value $U_{i}^{n}$, the value of the integral (3.1) on the interval $I$, etc. Figure 2 contains the description of a node.

The function $\bar{f}$ is an auxiliary function intoduced to calculate the integral (3.1). For all $\xi \in \mathbf{R}$, we define $\bar{f}^{\prime}(\xi)=\left|f^{\prime \prime}(\xi)\right|$. It follows that

$$
\int_{x_{i}^{n}}^{x_{i+1}^{n}}\left|U_{x x}^{n}\right|+\left|f^{\prime \prime}\left(U^{n}\right)\right|\left(U_{x}^{n}\right)^{2} d x=\frac{U_{i+1}^{n}-U_{i}^{n}}{h_{i}^{n}}\left(\bar{f}\left(U_{i+1}^{n}\right)-\bar{f}\left(U_{i}^{n}\right)\right),
$$

since $U_{x x}^{n}$ is 0 for all $x \in\left(x_{i}^{n}, x_{i+1}^{n}\right)$. For each minimal interval, we use (6.1) to calculate right.integral and left.integral; for each interior node, left.integral and right.integral are the values of this.integral for the left and right children of the node. For any node, we calculate this.integral by adding the difference of the left and right derivatives of $u$ at the meshpoint $x_{i}^{n}$ to the sum of left.integral and right.integral.

\section{Node:}

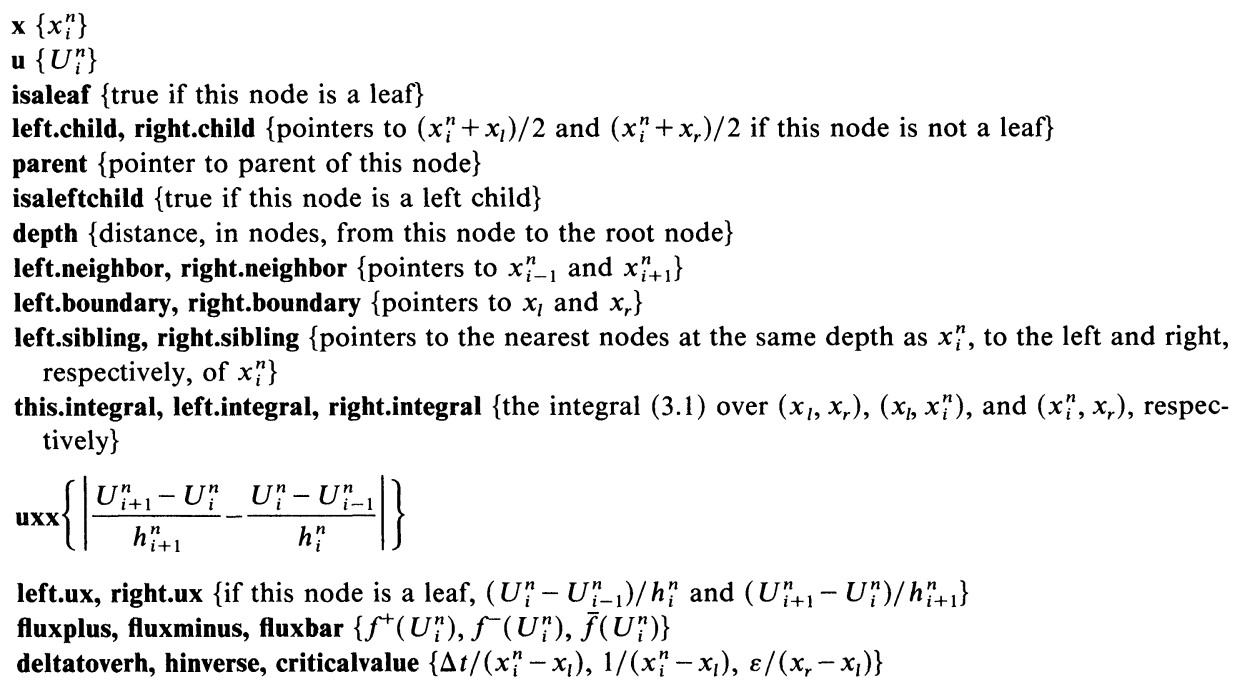
tively\}

$\mathbf{u x x}\left\{\left|\frac{U_{i+1}^{n}-U_{i}^{n}}{h_{i+1}^{n}}-\frac{U_{i}^{n}-U_{i-1}^{n}}{h_{i}^{n}}\right|\right\}$

left.ux, right.ux \{if this node is a leaf, $\left(U_{i}^{n}-U_{i-1}^{n}\right) / h_{i}^{n}$ and $\left.\left(U_{i+1}^{n}-U_{i}^{n}\right) / h_{i+1}^{n}\right\}$

fluxplus, fluxminus, fluxbar $\left\{f^{+}\left(U_{i}^{n}\right), f^{-}\left(U_{i}^{n}\right), \bar{f}\left(U_{i}^{n}\right)\right\}$

deltatoverh, hinverse, criticalvalue $\left\{\Delta t /\left(x_{i}^{n}-x_{l}\right), 1 /\left(x_{i}^{n}-x_{l}\right), \varepsilon /\left(x_{r}-x_{l}\right)\right\}$

FIG. 2. Definition of a node corresponding to $x_{i}^{n}$ in the interval $\left(x_{l}, x_{r}\right)$.

In each node we have included pointers and numerical variables whose values can be calculated from already available information. For example, in each leaf node $x_{i}^{n}$ we save $1 h_{i}^{n}$ and $\Delta t / h_{i}^{n}$. These values are needed often in the scheme, and change 
only when the mesh is changed. Our experience with the scheme has shown that, on average, less than one node is added to or subtracted from the mesh at each time step. Thus, using the saved values reduces execution time significantly. Because a new mesh is constructed at each timestep, we also require that the structural information necessary to derive a new mesh be readily available at each node. Again, extra storage reduces execution time. Since, for many problems, many fewer nodes are necessary to obtain a satisfactory error with this method than with standard first order methods, the extra storage requirements are not deemed critical.

The special nodes $a$ and $b$ are the left and right boundaries of $[a, b]$, the root node. In addition, supplemental meshpoints are added to the left and to the right of the interval $[a, b]$ so that the pointers left.sibling and right.sibling will not be nil for any node in the tree headed by $[a, b]$. The complete data structure is shown in Fig. 3. The lines in Fig. 3 illustrate only the children and sibling relationships of the nodes.

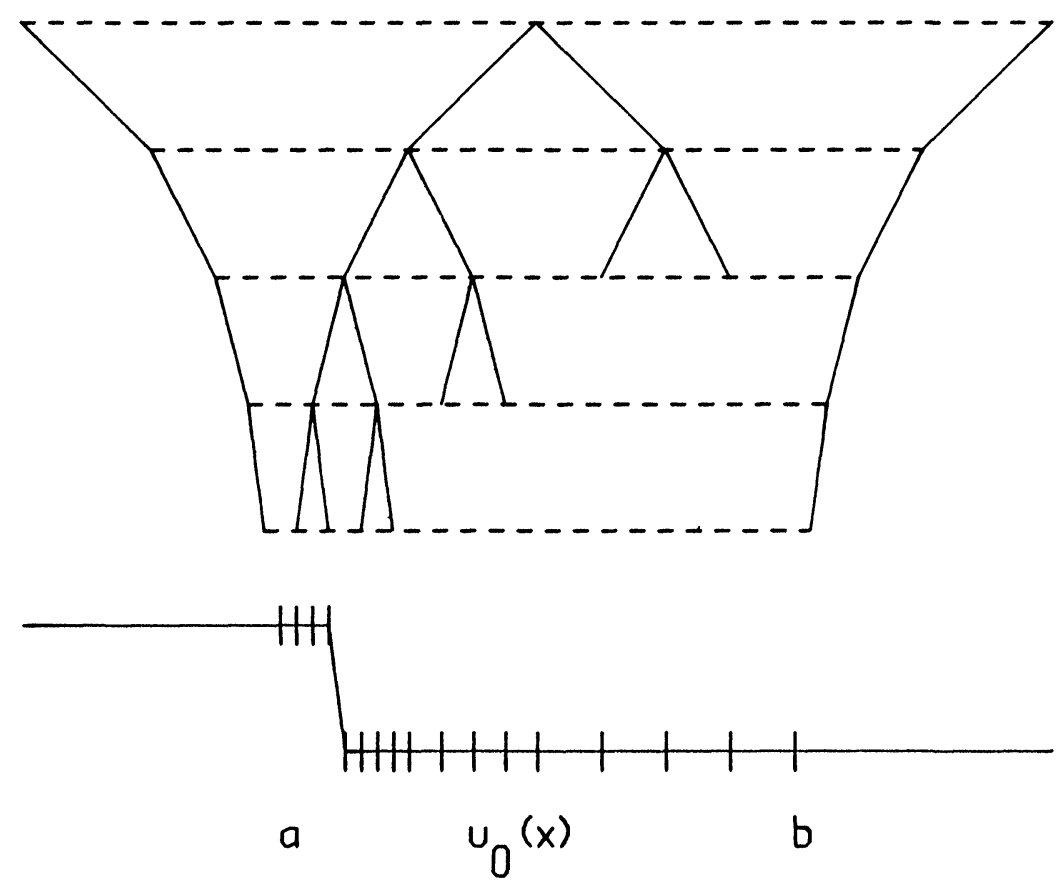

FIG. 3. Initial datum $u_{0}(x)$ with generated mesh (tic marks). The data structure, indicating sibling relationships (dashed lines) and parent-child relationships (solid lines), is drawn for this mesh. Each node in the tree corresponds to the meshpoint below it.

Figure 4 presents the procedure criteria that calculates the nodal values needed to build the new mesh. On each invocation, criteria calculates the nodal parameters for all nodes in the subtree headed by $p$. Indirection is indicated by a caret $\left(^{\wedge}\right)$. The with statement means that all unqualified nodal variables belong implicitly to the node to which the pointer " $p$ " points. For example, "right.neighbor .left.ux" refers to $p$ 's right.neighbor's left.ux. Each timestep, the nodal values for $a$ and $b$ are calculated first, and then criteria(root) is called. The following lemma outlines a proof that the procedure is correct.

LEMma 6.1. Criteria ( $p)$ calculates the values of right.integral, left.integral, uxx, and this.integral for all nodes in the subtree headed by $p$. Furthermore, criteria $(p)$ calculates left.ux and right.ux for all leaves in the subtree headed by $p$. 


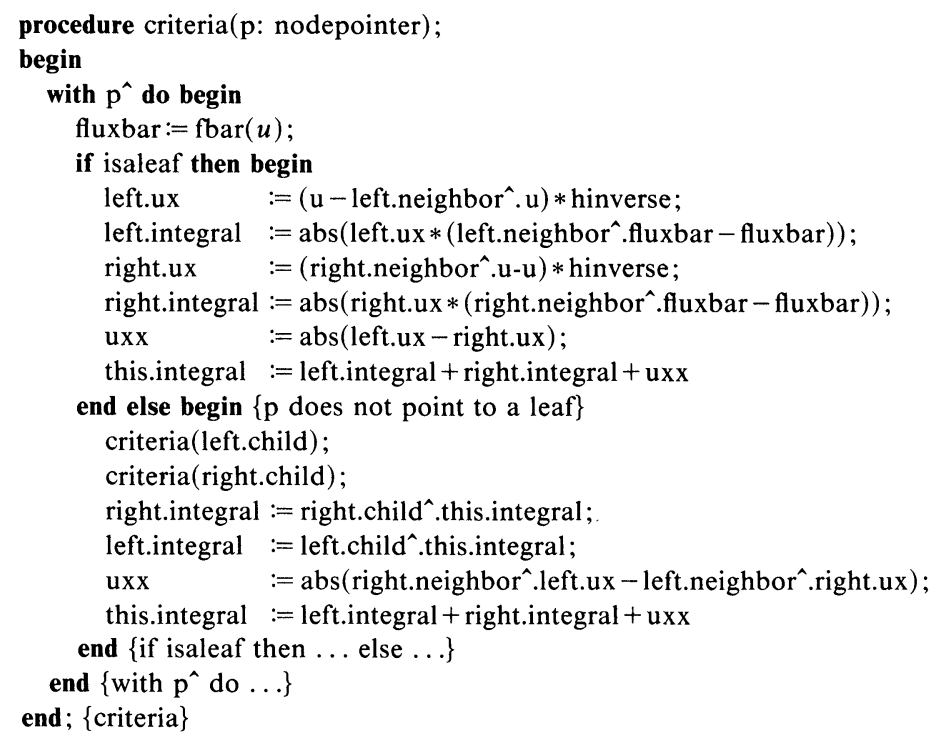

FIG. 4. Procedure for calculating the integral (3.1).

Proof. First one shows that $\bar{f}\left(U_{i}^{n}\right)$ is calculated for the left and right boundary points of $p$ before criteria $(p)$ is called. This easily proved by noting that it is true initially for the root node, and that if it is true for $p$ 's parent, then it is true for $p$. The proof of the lemma then follows by induction on the height of the subtree headed by $p$.

To construct the mesh $\left\{x_{i}^{n+1}\right\}$ from $\left\{x_{i}^{n}\right\}$, points are first added to $\left\{x_{i}^{n}\right\}$ at the leaves to obtain $\left\{x_{i}^{n}\right\} \cup\left\{x_{i}^{n+1}\right\}$. In the second pass, certain subtrees of interior nodes are removed from the union to leave only $\left\{x_{i}^{n+1}\right\}$. Since the set of points that are tested on each pass are for the most part disjoint (only nodes with newly added children are tested twice), this two pass algorithm does not add too much to the arithmetic complexity of the scheme. A one pass algorithm could surely be devised.

It remains to find a suitable way to calculate (3.1) from the information stored in the nodes. An argument similar to that in lemma 3.1 shows that, in any mesh constructed using Algorithm M, a node's parent will always have adjacent left and right siblings. If $\left\{x_{i}^{n}\right\} \cup\left\{x_{i}^{n+1}\right\}$ is formed from $\left\{x_{i}^{n}\right\}$ by adding all meshpoints of one depth before proceeding to the next, then this property also holds for all meshes intermediate to $\left\{x_{i}^{n}\right\}$ and $\left\{x_{i}^{n}\right\} \cup\left\{x_{i}^{n+1}\right\}$. Thus, we add or remove nodes in a breadth first ordering, and calculate (3.1) for an interval that is a left child, for example, by adding $p^{\wedge}$.parent ${ }^{\wedge}$.this.integral $p^{\wedge}$.parent ${ }^{\wedge}$.left.sibling .right.integral, and $p^{\wedge}$.left.boundary^.uxx. Fig. 5 illustrates the calculation. A similar expression holds for nodes that are right children.

The above calculation is the reason that each admissible interval has a meshpoint at its center, and that the integral (3.1) is calculated over the left and right halves of each admissible interval.

Note that our algorithm removes unnecessary subtrees at once, instead of removing one meshpoint at a time. In practice, however, we have not observed the removal of any but the trivial subtrees consisting of only one node.

We use a simple and efficient memory management scheme, which may, however, fragment memory usage in a virtual memory system. When a node is no longer needed and is removed from the tree, it is appended to a free list of nodes. When a node is 


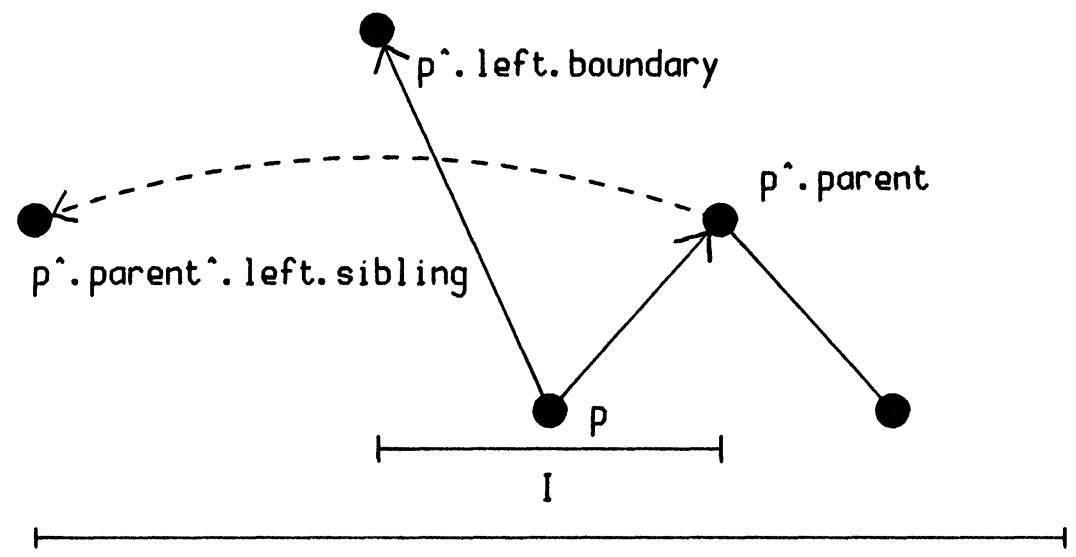

$3 I$

FIG. 5. The calculation of the integral (3.1).

needed for addition to the tree, it is taken from the free list if the list is not empty. If the free list is empty, a call to the operating system allocates enough storage for the new node.

The complexity of the scheme can be measured by counting the number of floating point operations that are done, on average, for each meshpoint $x_{i}^{n}$ at each time $t^{n}$. More operations are performed for leaf nodes than for interior nodes. We assume that an evaluation of $f^{+}, f^{-}$, or $\bar{f}$ requires two additions (or subtractions), two multiplications, and two comparisons. This would be the case, for example, if these functions were defined as piecewise quadratic functions over four intervals. Our assumptions yield

Complexity per meshpoint $=17$ additions +9.5 multiplications +7 comparisons.

This may be compared with a complexity estimate of 8 additions, 4 multiplications, and 4 comparisons for a careful implementation of a uniform mesh algorithm with the same spatial difference operator and $\Delta t=h$. If an arbitrary variable spaced mesh is chosen every timestep, the standard algorithm will require 10 additions, 5 multiplications, 2 divisions, and 4 comparisons per meshpoint. This does not include whatever calculations are necessary to choose the mesh. Thus, the special placement of the meshpoints in our algorithm no more than doubles the work per meshpoint.

Nonarithmetic operations must be included in any complexity estimate of these algorithms. It is more difficult to quantify this nonarithmetic complexity, what may be considered "overhead." We give empirical results in the next section that show that the nonarithmetic overhead is about the same for the fixed and adaptive mesh algorithms.

A more serious difficulty in comparing the efficiency of these algorithms is that the fixed mesh algorithm converges at different rates for differing fluxes $f$. These convergence rates depend on whether the flux is uniformly convex, or linear, or possibly whether it has a point of inflection; the rates also depend on the smoothness of the solution. While some of these results are well known, we present them all in the next section. We will find that for the problems for which the fixed mesh algorithm performs relatively well, the new algorithm compares poorly. The problems for which the fixed mesh algorithm performs poorly, however, are solved with surprising success by our algorithm. 
7. Computational results. The Pascal implementation of our algorithm was run on two Digital Equipment Corporation VAX 11/780 computers with floating point accelerators under the VMS 2.5 and Berkeley Unix 4.1 BSD operating systems. Both programs use double precision (64 bit) floating point numbers. We only needed to change real constants from double precision to single precision notation to move the program from VMS to Unix; no other changes were needed.

We chose computational examples to highlight the strengths of our method as well as point out directions for improvements. The biggest omission was of problems with smooth solutions. (We did not implement the algorithm for smooth solutions given in $\S 5$ ). It may be shown that for monotone uniform grid methods, the work expended to achieve an accuracy of $\delta$ at time $T$ is proportional to $\delta^{-2}$. When given a problem with a smooth solution, the present implementation of our method will achieve the same accuracy, but will take timesteps that are much smaller than the average mesh spacing. In fact, we show in the discussion of the second numerical experiment that the work for our method will be proportional to $\delta^{-3}$. This shortcoming will be taken up in a broader context below. We have compared the methods for problems with shocks, contact discontinuities, and expansion waves following shocks. These comparisons lead us to believe that our mehod is superior to fixed mesh monotone methods when the discontinuities in the solutions of $(\mathrm{C})$ are smeared across more than a fixed number of mesh intervals by the monotone schemes.

In these examples, our method is compared with a finite difference scheme with a fixed, uniform, spatial grid. This scheme's difference operator is

$$
\frac{U_{i}^{n+1}-U_{i}^{n}}{\Delta t}+\frac{f^{+}\left(U_{i}^{n}\right)-f^{+}\left(U_{i-1}^{n}\right)}{h}+\frac{f^{-}\left(U_{i+1}^{n}\right)-f^{-}\left(U_{i}^{n}\right)}{h}=0 .
$$

Always $\Delta t=h$ to avoid any divisions or multiplications in this part of the algorithm. A careful implementation of the comparison scheme uses only two function evaluations, three subtractions, and one addition per meshpoint per timestep.

For all examples but the sixth, $f^{-}=0$, so that each difference operator reduces to the standard upwind-difference scheme. The initial values $U_{i}^{0}$ were chosen to interpolate the initial data $u_{0}$ at the points $i h$. The solution of the fixed mesh method was interpreted as a piecewise linear function taking on the values $U_{i}^{n}$ at the points $(i h, n \Delta t)$. The difference in $L^{1}[a, b]$ between the approximate solution and the piecewise linear interpolant of the true solution $u(x, t)$ was recorded as the error of the both schemes.

Table 1 presents the fluxes $f$ and the initial and final values of $u$ for the computational experiments. Table 2 contains the errors and execution times, respectively, corresponding to various values of $\Delta t$. For the purposes of comparing the methods, the parameter $\delta$, a measure of the average mesh spacing where the solution is smooth, is introduced. The parameter $\delta$ has the value $\sqrt{\Delta t}$ for the adaptive mesh method, and $\Delta t$ for the fixed mesh method. Table 3 tabulates an expression of the error as error $=C \tau^{\alpha}$ and error $=C \delta^{\alpha}$ for each test, where $\tau$ denotes the CPU time for the run.

The CPU times in Table 2 are the "user" times reported by the Unix operating system. The "system" time, which measures the time spent by the operating system to service the program, was not included. The system time varied greatly, depending on the system usage, so it was not deemed a reliable measure of the methods' resource needs. The user times corresponded well with the CPU times measured on the VMS operating system on a lightly loaded machine. The error decay rates in Table 3 are based on a log-log least squares fitting of the data. They were calculated from the data in Table 2, but with the full accuracy of the data, which is truncated in Table 2. 
TABLE 1

Flux, initial and final values of $u(x, t)$.

\begin{tabular}{|c|c|c|c|c|}
\hline & $f(u)$ & & $u_{0}(x)$ & $u(x, 4)$ \\
\hline 1 & $\left(u+u^{2}\right) / 4$ & & $\begin{cases}1 & \text { if } x \leqq 1 \\
0 & \text { if } x>1\end{cases}$ & $\begin{cases}1 & \text { if } x \leqq 3 \\
0 & \text { if } x>3\end{cases}$ \\
\hline 2 & $\left(u+u^{2}\right) / 4$ & & $\begin{cases}x-1 & \text { if } 1 \leqq x \leqq 2 \\
3-x & \text { if } 2 \leqq x \leqq 3 \\
0 & \text { otherwise }\end{cases}$ & $\begin{cases}(x-2) / 3 & \text { if } 2 \leqq x<2+\sqrt{6} \\
0 & \text { otherwise }\end{cases}$ \\
\hline 3 & $u / 2$ & & $u(x+2,4)$ & $\begin{cases}2-4|x-3| & \text { if }|x-3| \leqq \frac{1}{2} \\
0 & \text { otherwise }\end{cases}$ \\
\hline 4 & $u / 2$ & & $\begin{cases}1 & \text { if } x \leqq 1 \\
0 & \text { if } x>1\end{cases}$ & $\begin{cases}1 & \text { if } x \leqq 3 \\
0 & \text { if } x>3\end{cases}$ \\
\hline 5 & $\begin{cases}1-(1-u)^{2} & \text { if } u \geqq \\
u^{2} & \text { if } u \leqq\end{cases}$ & & $\begin{cases}1 / \sqrt{2} & \text { if } x \leqq 1 \\
0 & \text { otherwise }\end{cases}$ & $\begin{cases}1 / \sqrt{2} & \text { if } x \leqq 9-4 \sqrt{2} \\
0 & \text { otherwise. }\end{cases}$ \\
\hline 6 & $\left\{\begin{array}{l}1-(1-u)^{2} \\
-2 u^{3}+3 u^{2}-u / 2\end{array}\right.$ & $\begin{array}{l}\text { if } u \geqq \frac{1}{2} \\
\text { if } u \leqq \frac{1}{2}\end{array}$ & $\begin{cases}1 & \text { if } x \leqq 1 \\
0 & \text { otherwise }\end{cases}$ & $\begin{cases}1 & \text { if } x \leqq 1 \\
1-(x-1) / 8 & \text { if } 1 \leqq x \leqq 9-4 \sqrt{2} \\
0 & \text { otherwise. }\end{cases}$ \\
\hline
\end{tabular}

TABLE 2

Computational results.

\begin{tabular}{|c|c|c|c|c|c|c|c|c|}
\hline \multirow{3}{*}{$\begin{array}{l}\delta^{-1} \\
2\end{array}$} & \multicolumn{4}{|c|}{ Test 1 . } & \multicolumn{4}{|c|}{ Test 2.} \\
\hline & \multicolumn{2}{|c|}{ Adaptive mesh } & \multicolumn{2}{|c|}{ Fixed mesh } & \multicolumn{2}{|c|}{ Adaptive mesh } & \multicolumn{2}{|c|}{ Fixed mesh } \\
\hline & $1.252-1$ & 0.12 & $2.258-1$ & 0.02 & $2.468-1$ & 0.13 & $3.419-1$ & 0.02 \\
\hline 4 & $3.237-2$ & 0.88 & $1.252-1$ & 0.07 & $1.174-1$ & 1.18 & $2.079-1$ & 0.07 \\
\hline 8 & $8.093-5$ & 5.25 & $6.456-2$ & 0.27 & $5.449-2$ & 8.88 & $1.119-1$ & 0.28 \\
\hline 16 & $2.023-3$ & 26.33 & $3.237-2$ & 1.07 & $3.169-2$ & 58.85 & $6.276-2$ & 1.07 \\
\hline 32 & $5.058-4$ & 132.65 & $1.618-2$ & 4.37 & $1.771-2$ & 383.15 & $2.852-2$ & 4.48 \\
\hline 64 & $1.264-4$ & 625.68 & $8.093-3$ & 17.65 & $9.547-3$ & 2560.93 & $1.308-2$ & 17.22 \\
\hline 128 & $3.161-5$ & 2988.28 & $4.046-3$ & 71.25 & $5.129-3$ & 17678.77 & $6.782-3$ & 69.95 \\
\hline \multirow[t]{2}{*}{$\delta^{-1}$} & \multicolumn{4}{|c|}{ Test 3.} & \multicolumn{4}{|c|}{ Test 4.} \\
\hline & \multicolumn{2}{|c|}{ Adaptive mesh } & \multicolumn{2}{|c|}{ Fixed mesh } & \multicolumn{2}{|c|}{ Adaptive mesh } & \multicolumn{2}{|c|}{ Fixed mesh } \\
\hline 2 & $8.727-1$ & 0.12 & $1.116-0$ & 0.02 & $3.611-1$ & 0.10 & $4.560-1$ & 0.02 \\
\hline 4 & $4.010-1$ & 1.22 & $8.627-1$ & 0.05 & $1.904-1$ & 0.88 & $3.425-1$ & 0.07 \\
\hline 8 & $1.374-1$ & 10.62 & $6.139-1$ & 0.25 & $1.022-1$ & 6.45 & $2.530-1$ & 0.23 \\
\hline 16 & $5.532-2$ & 73.72 & $3.954-1$ & 1.00 & $5.429-2$ & 42.57 & $1.846-1$ & 1.00 \\
\hline 32 & $2.747-2$ & 493.65 & $2.302-1$ & 4.02 & $2.942-2$ & 299.60 & $1.335-1$ & 3.98 \\
\hline 64 & $1.348-2$ & 3168.97 & $1.229-1$ & 16.07 & $1.532-2$ & 2235.78 & $9.592-2$ & 16.00 \\
\hline 128 & $6.091-3$ & 20316.55 & $6.243-2$ & 64.45 & $7.898-3$ & 17124.27 & $6.860-2$ & 64.40 \\
\hline \multirow[t]{2}{*}{$\delta^{-1}$} & \multicolumn{4}{|c|}{ Test 5.} & \multicolumn{4}{|c|}{ Test 6.} \\
\hline & \multicolumn{2}{|c|}{ Adaptive mesh } & \multicolumn{2}{|c|}{ Fixed mesh } & \multicolumn{2}{|c|}{ Adaptive mesh } & \multicolumn{2}{|c|}{ Fixed mesh } \\
\hline 2 & $1.091-1$ & 0.12 & $2.803-1$ & 0.02 & $2.018-1$ & 0.12 & $4.234-1$ & 0.02 \\
\hline 4 & $4.051-2$ & 0.92 & $1.088-1$ & 0.10 & $9.276-2$ & 0.92 & $1.969-1$ & 0.07 \\
\hline 8 & $1.863-2$ & 5.27 & $8.775-2$ & 0.33 & $4.633-2$ & 6.45 & $1.477-1$ & 0.30 \\
\hline 16 & $6.475-3$ & 29.25 & $3.872-2$ & 1.20 & $2.174-2$ & 41.32 & $7.532-2$ & 1.18 \\
\hline 32 & $2.404-3$ & 154.78 & $3.043-2$ & 4.68 & $1.069-2$ & 253.40 & $5.345-2$ & 4.68 \\
\hline 64 & $1.222-3$ & 858.48 & $1.614-2$ & 19.08 & $5.440-3$ & 1594.37 & $2.983-2$ & 18.87 \\
\hline 128 & $6.676-4$ & 4927.42 & $8.462-3$ & 71.83 & $2.716-3$ & 10267.05 & $1.644-2$ & 75.70 \\
\hline
\end{tabular}


TABLE 3

Error decay rates.

\begin{tabular}{c|ll|ll}
\hline \multicolumn{2}{c}{ Test } & \multicolumn{2}{c}{ Adaptive mesh } & \multicolumn{2}{c}{ Fixed mesh } \\
\hline 1 & $e=.027 \tau^{-.826}$ & $e=.508 \delta^{1.995}$ & $e=.033 \tau^{-.493}$ & $e=.472 \delta^{.975}$ \\
2 & $e=.121 \tau^{-.326}$ & $e=.419 \delta^{.915}$ & $e=.057 \tau^{-.487}$ & $e=.770 \delta^{.961}$ \\
3 & $e=.377 \tau^{-.418}$ & $e=1.864 \delta^{1.200}$ & $e=.506 \tau^{-.499 *}$ & $e=.246 \delta^{.995 *}$ \\
4 & $e=.179 \tau^{-.319}$ & $e=.684 \delta^{.915}$ & $e=.183 \tau^{-.233}$ & $e=.643 \delta^{.457}$ \\
5 & $e=.037 \tau^{-.481 *}$ & $e=.213 \delta^{1.207 *}$ & $e=.050 \tau^{-.440 *}$ & $e=.488 \delta^{.864 *}$ \\
6 & $e=.091 \tau^{-.381}$ & $e=.397 \delta^{1.034}$ & $e=.087 \tau^{-.376}$ & $e=.652 \delta^{.749}$ \\
\hline
\end{tabular}

* See text.

In general, the computational tests show that solutions of the adaptive numerical method converge to the solution of the conservation law at least to within order $\Delta t^{1 / 2}$. This implies, in particular, that shock speeds are correct to within that order. The computations also show that the mass balance errors are within the bounds predicted by Theorems 4.4 and 4.5 ; it is also true, but not reported in the tables, that the hypotheses of these theorems were satisfied by the experiments. Furthermore, it is interesting to note that for Riemann problems with uniformly convex fluxes or linear fluxes, the shock speed and mass balance errors were of order $\Delta t$.

Test 1 presents our method in the best light. This is a Riemann problem with a quadratic nonlinearity, similar to that of Burgers' equation, that keeps the shock width to within a few of the small $(O(\Delta t))$ mesh intervals. The shock speed is also correct to within one small mesh interval. At the same time, the numerical solution is nearly constant outside the shock region, so only $O(-\log (\Delta t))$ meshpoints are necessary to accurately represent the solution. The result is an error of $O(\Delta t)$ with a computational complexity of $O(-\log (\Delta t) / \Delta t)$.

Test 2 is perhaps the worst comparison between the fixed mesh and adaptive mesh methods. The adaptive method gives no more than $O\left(\varepsilon^{1 / 2}\right)$ accuracy in the regions where the solution is smooth. To do so, it uses $O\left(\varepsilon^{-1 / 2}\right)$ meshpoints. Coupled with a time step of $O(\varepsilon)$, we achieve a complexity bound of $O\left(\varepsilon^{-3 / 2}\right)$, a decidedly inferior result when compared with the fixed mesh. This predicament strongly suggests a method where the local timesteps are proportional to the local meshsize. Such methods have been used by Oliger [21], Bolstad [2], Berger [1], and others (see references in [14]). With such a scheme, the error will still be $O\left(\varepsilon^{1 / 2}\right)$, but the complexity of the scheme will be reduced to $O(\varepsilon)$. This is the same relationship between error size and complexity that applies for the fixed mesh method, which does suprisingly well for this problem.

When applied to the third test problem, the fixed mesh method behaves in a different way when the meshsize is small than when it is large. This is because of the large second derivatives in the solution. The fixed mesh method exhibits an error of order $\Delta t^{\alpha}$, with $\alpha$ decidedly less than one, when the meshsize is large (greater than $1 / 32$ ). When the meshsize is small, however, the mesh can adequately resolve the large gradients and the error is of order $\Delta t$. Because we are mainly interested in exhibiting "asymptotic" error rates, we have computed the error for the fixed mesh method for $\Delta t$ as small as $1 / 1024$, and our error decay rates in Table 3 are derived from the smaller timesteps.

Test 4 was the problem that motivated the design of the adaptive method. It corresponds to a contact discontinuity in gas dynamics. The fixed mesh method has an accuracy of order $\Delta t^{1 / 2}$, because it smears the discontinuity over $O\left(\Delta t^{-1 / 2}\right)$ intervals. The time complexity of the fixed mesh method is $O\left(\Delta t^{-2}\right)$, so the error of the method 
is of order $\tau^{-1 / 4}$. The adaptive method puts $O\left(\Delta t^{-1 / 2}\right)$ intervals of size $\Delta t$ near the discontinuity, and the same number of size $\Delta t^{1 / 2}$ away from it, while achieving an accuracy of $\Delta t^{1 / 2}$. Because there are $O\left(\Delta t^{-1}\right)$ timesteps, the complexity of the adaptive scheme is of order $\Delta t^{-3 / 2}$; the error is of order $\tau^{-1 / 3}$. In other words, if one wants an error of order $\varepsilon$, it takes order $\varepsilon^{-4}$ work for the fixed method, and order $\varepsilon^{-3}$ work for the adaptive method.

The solutions of Tests 5 and 6 exhibit behavior similar to the solutions of the Buckley-Leverett equation, which is used as a model in petroleum reservoir simulation (see Douglas and Wheeler [9]). In general, solutions of these equations consist of a shock followed by a smooth expansion wave. Test 5 examines how well the shock itself is tracked, while Test 6 investigates the complete system, shock and expansion wave.

In Test 5 , the fluid behind the discontinuity is moving at the same speed as the discontinuity, whereas the fluid in front of the shock is moving at a slower speed. One may suspect, therefore, that behind the shock the adaptive scheme will act as for a linear flux, as in Test 4, and before the shock it will act as for a uniformly convex flux, as in Test 1 . This is indeed the case; here the lack of conservation is most severely felt. The following description of the solution seems to hold as the stepsize is reduced. Numerical diffusion erodes the wave front behind the shock, as for the linear problem, while the nonlinearity at the front of the shock holds the discontinuity sharp at $u=0$. The height of the wave immediately behind the front is reduced by a factor of $O\left(\Delta t^{1 / 2}\right)$, and since the shock speed depends on the height of the wave, the shock speed is reduced by $O\left(\Delta t^{1 / 2}\right)$, thereby incurring a mass balance error of $O\left(\Delta t^{1 / 2}\right)$. There are $O\left(\Delta t^{-1 / 2}\right)$ minimal intervals of width less than $\varepsilon$ behind the shock, and $O\left(\Delta t^{-1 / 2}\right)$ minimal intervals of width greater than $\varepsilon$ behind the small intervals, as for the linear problem. These errors for the mass balance and shock speed are exactly of the order predicted by Theorem 4.5 if the assumptions of Theorem 4.5, which seem completely plausible and which have been observed for the values of $\Delta t$ used here, are accepted. The conservation of the fixed mesh scheme ensures that its shock speed is correct, and that since the shock speed is greater than the downwind fluid velocity, the shock transition is sharp, and the error is $O(\Delta t)$.

The error rates reported in Table 3 for Test 5 were calculated using the values of $\delta$ down to $1 / 256$ for the adaptive method and values of $\delta$ down to $1 / 1024$ for the fixed mesh problem. The calculated error is not a very smooth function of $\delta$, because the final shock position, $9-4 \sqrt{2}$, never lies on a meshpoint; the error depends not only on how well the shock speed is approximated, but also on how closely one can approximate the final shock position on the mesh. The description of the structure of the adaptive solution was developed using the solutions with the four smallest meshsizes, for which it seemed that the asymptotic regime was reached.

Test 6 incorporates an expansion wave that follows the shock. The fixed mesh solution exhibits an accuracy of order $\Delta t^{3 / 4}$, instead of $\Delta t$ for the problem with only the shock. The tests indicate that the relationship between complexity and error is the same for the adaptive method and the fixed mesh method. Changing the adaptive method to use locally varying timesteps as well as mesh spacing would greatly decrease the CPU time for this problem.

The results of Tests 1 through 6 suggest that our new method is effective when discontinuities in the solutions are smeared across many mesh intervals by the fixed mesh method.

An alternate approach to developing an adaptive mesh method is presented in [9]. There, the motivation is to use an implicit scheme with a large timestep and to 
refine the mesh near roughness in the solution. Unfortunately, this strategy does not asymptotically decrease the error; heuristically, this is because one cannot drag a shock or discontinuity across many meshpoints in one timestep without smearing the discontinuity. This effect is illustrated in Table 4 for two problems, each of which has the solution $u(x)=\frac{1}{2}(1-\operatorname{sgn}(x-t / 2))$. The fluxes are $f(u)=\frac{1}{2} u$ and $f(u)=\frac{1}{4}\left(u+u^{2}\right)$, respectively. By running each problem with $h$, the mesh spacing, equal to $\Delta t$ and $\Delta t^{2}$, our choice is not to adapt the mesh, but to use a uniformly fine mesh everywhere, so that our results do not depend on any particular mesh refinement strategy. The error for the first problem is of order $\Delta t^{1 / 2}$ and the error for the second problem is of order $\Delta t$, regardless of the choice of mesh. Thus, it appears that spatial mesh refinement, without a corresponding refinement of the temporal increments, is not effective in solving these problems.

TABLE 4

Errors using implicit upwind-difference scheme.

\begin{tabular}{ccccc}
\hline \multicolumn{2}{c}{ st } & \multicolumn{2}{c}{ Test 1. } & \multicolumn{2}{c}{ Test 2. } \\
& $h=\Delta t^{2}$ & $h=\Delta t^{2}$ & $h=\Delta t$ \\
\hline 0.500000 & $7.8550 e-01$ & $9.5360 e-01$ & $5.1312 e-01$ & $6.7648 e-01$ \\
0.250000 & $4.8564 e-01$ & $6.8264 e-01$ & $2.3904 e-01$ & $4.1525 e-01$ \\
0.125000 & $3.1453 e-01$ & $4.8564 e-01$ & $1.0772 e-01$ & $2.3833 e-01$ \\
0.062500 & $2.1129 e-01$ & $3.4444 e-01$ & $4.9128 e-02$ & $1.2741 e-01$ \\
0.031250 & $1.4529 e-01$ & $2.4393 e-01$ & $2.3225 e-02$ & $6.4799 e-02$ \\
0.015625 & $1.0124 e-01$ & $1.7261 e-01$ & $1.1274 e-02$ & $3.2443 e-02$ \\
\hline
\end{tabular}

In the previous section, we showed that the arithmetic complexity of the adaptive mesh algorithm was no more than twice that of the fixed mesh algorithm per meshpoint per timestep. The algorithms differ greatly in their implementations, however. The fixed mesh algorithm is almost trivial to implement, while the adaptive method uses sophisticated data structures and pointer manipulation. We therefore set out to quantify the amount of nonarithmetic overhead in each method.

We proposed to measure the proportion of the CPU time spent in arithmetic computations as compared with nonarithmetic computations for both implementations. We had available two VAX's running identical software, one of which did not have a floating point accelerator (FPA). A simple program consisting mainly of an equal number of nontrivial memory to register floating point additions and multiplications was run and timed on both machines. A speedup of a factor of five was observed on the machine with the FPA. We then compared the CPU times for the same implementations of the two algorithms on both machines. The ratios of the CPU times are given in Table 5. Assuming that only floating point operations were speeded up by the FPA

TABLE 5

Nonarithmetic overhead.

\begin{tabular}{ccccccc} 
& \multicolumn{3}{c}{ Adaptive mesh } & \multicolumn{2}{c}{ Fixed mesh } \\
Test & $\begin{array}{c}\text { CPU time } \\
\text { ratio }\end{array}$ & FPA & No FPA & CPU time & \multicolumn{2}{c}{ Overhead } \\
ratio & FPA & No FPA \\
\hline 4 & 0.728 & $91 \%$ & $66 \%$ & 0.678 & $88 \%$ & $60 \%$ \\
6 & 0.699 & $89 \%$ & $63 \%$ & 0.715 & $90 \%$ & $64 \%$ \\
\hline
\end{tabular}


(a faulty assumption, for some integer arithmetic is also faster), we calculated the fraction of time spent in nonarithmetic operations by both programs on both machines. For Test 6 , with nontrivial $f^{+}, f^{-}$, and $\bar{f}$, around $90 \%$ of the time spent on nonarithmetic operations on the machine with the FPA for both problems. The figure is similar to Test 4 , with trivial $f^{+}, f^{-}$, and $\bar{f}$.

To discover the effects of the Pascal compiler, we implemented the fixed mesh algorithm in FORTRAN and ran this program on the machine with he FPA. For some reason, the FORTRAN compiler generated much superior code for index calculations. Function calls were slightly cheaper because the FORTRAN program, not being block structured, did not maintain a "display" of the currently accessible data areas. The computation time of the FORTRAN program was $80 \%$ of its Pascal counterpart. This still leaves us with an overhead figure of about $87 \%$. These tests indicate that the overhead is similar, and large, for each algorithm.

Acknowledgments. This work benefited greatly from a number of suggestions made by Jerry Bona, Todd Dupont, and the referees.

\section{REFERENCES}

[1] M. BERGER, Adaptive mesh refinement for hyperbolic partial differential equations, dissertation, Stanford Computer Science Report STAN-CS-82-924, Stanford Univ., Stanford, CA, 1982.

[2] J. H. BolstAD, An adaptive finite difference method for hyperbolic systems in one space dimension, dissertation, Lawrence Berkeley Lab. LBL-13287 (STAN-CS-82-899), Stanford, CA, 1982.

[3] C. DE BOOR, Good approximation by splines with variable knots, in Spline Functions and Approximation Theory, A. Meir and A. Sharma, eds., ISNM 21, Birkhauser Verlag, Berlin, 1973, pp. 57-72.

[4] - Good approximation by splines with variable knots II, in Lecture Notes in Mathematics 363, Springer-Verlag, Berlin, 1974, pp. 12-20.

[5] R. COURANT, E. ISAACSON AND M. REES, On the solution of nonlinear hyperbolic differential equations by finite differences, Comm. Pure Appl. Math., 5 (1952), pp. 243-255.

[6] M. G. CRANDAll AND A. MAJDA, Monotone difference approximations for scalar conservation laws, Math. Comp., 34 (1980), pp. 1-21.

[7] S. F. DAvis AND J. E. Flaherty, An adaptive finite element method for initial-value problems for partial differential equations, SIAM J. Sci. Stat. Comput., 3 (1982), pp. 6-28.

[8] J. Douglas JR., Simulation of a linear waterflood, in Free Boundary Problems, Proceedings of a seminar held in Pavia, Sept.-Oct. 1979, Vol. II, Institute Nazionale de Alta Matematica Francesco Severi, Rome, 1980.

[9] J. Douglas JR. AND M. F. WheEler, Implicit, time-dependent variable grid finite difference methods for the approximation of a linear waterflood, Math. Comp., 40 (1983), pp. 107-122.

[10] Todd Dupont, Mesh modification for evolution equations, Math. Comp., 39 (1982), pp. 85-107.

[11] B. ENQUIST AND S. OSHER, Stable and entropy satisfying approximations for transonic flow calculations, Math. Comp., 34 (1980), pp. 45-75.

[12] D. B. GANNON, Self adaptive methods for parabolic partial differential equations, U. of Illinois, Computer Science Dept. Rep. UIUCDCS-R-80-1020, Univ. Illinois, Champaign-Urbana.

[13] A. HARTEN, J. M. HyMAN AND P. D. LAX, On finite difference approximations and entropy conditions for shocks, Comm. Pure Appl. Math., 29 (1976), pp. 297-322.

[14] G. W. Hedstrom AND G. H. Rodrigue, Adaptive-grid methods for time-dependent partial differential equations, in Multigrid Methods, W. Hackbusch and U. Trottenberg, eds., Springer-Verlag, Berlin, 1982, pp. 474-484.

[15] D. KNuth, The Art of Computer Programming, 1, 2nd ed., Addison-Wesley, Reading, MA, 1973.

[16] S. N. KRUZKOv, First order quasilinear equations with several space variables, Math. USSR Sb., 10 (1970), pp. 217-243.

[17] N. N. KuZnetsov, Accuracy of some approximate methods for computing the weak solutions of a first-order quasi-linear equation, USSR Comp. Math. and Math. Phys., 16, 6 (1976), pp. 105-119.

[18] R. LEVEQUE, Large time step shock-capturing trechniques for scalar conservation laws, SIAM J. Numer. Anal., 19 (1982), pp. 1091-1109.

[19] B. J. LUCIER, On nonlocal monotone difference methods for scalar conservations laws, to appear. 
[20] K. Miller, Moving finite elements, Part II, SIAM J. Numer. Anal., 18 (1981), pp. 1019-1057.

[21] J. Oliger, Approximate methods for atmospheric and oceanographic circulation problems, in Lecture Notes in Physics 91, R. Glowinski and J. Lions, eds., Springer-Verlag, Berlin, 1979, pp. 171-184.

[22] R. SANDERS, On convergence of monotone finite difference schemes with variable spatial differencing, Math. Comp., 40 (1983), pp. 91-106.

[23] B. VAN LEER, Towards the ultimate conservative difference scheme. V. A second-order sequel to Godunov's method, J. Comp. Phys. 32 (1979), pp. 101-136. 